\title{
Laplacian growth and Whitham equations of soliton theory
}

\author{
I. Krichever $^{*} \quad$ M. Mineev-Weinstein ${ }^{\dagger} \quad$ P. Wiegmann ${ }^{\ddagger}$ \\ A. Zabrodin $\S$
}

October 2003

\begin{abstract}
The Laplacian growth (the Hele-Shaw problem) of multiply-connected domains in the case of zero surface tension is proven to be equivalent to an integrable system of Whitham equations known in soliton theory. The Whitham equations describe slowly modulated periodic solutions of integrable hierarchies of nonlinear differential equations. Through this connection the Laplacian growth is understood as a flow in the moduli space of Riemann surfaces.
\end{abstract}

\footnotetext{
*Department of Mathematics, Columbia University, New York, USA, Landau Institute and ITEP, Moscow, Russia

${ }^{\dagger}$ Los Alamos National Laboratory, MS-P365, Los Alamos, NM 87545, USA

${ }_{\ddagger}^{\ddagger}$ James Frank Institute and Enrico Fermi Institute of the University of Chicago, 5640 S.Ellis Avenue, Chicago, IL 60637, USA and Landau Institute for Theoretical Physics, Moscow, Russia

${ }^{\S}$ CNLS, LANL, Los Alamos, NM 87545, USA, and Institute of Biochemical Physics, 4 Kosygina st., 119991, Moscow, Russia and ITEP, 25 B.Cheremushkinskaya, 117259, Moscow, Russia
} 


\section{Introduction}

It is not uncommon that nonlinear differential equations which possess an integrable structure emerge in important problems of hydrodynamics [1]. The Korteweg de Vries equation describing nonlinear waves in dispersive media is perhaps the most familiar example.

In recent years, integrable structures were found in another class of hydrodynamics problems leading to a pattern formation in a regime far from equilibrium [2]. Growth problems of this type are unified by the name Laplacian growth. In this paper, we further develop a link between growth processes and soliton theory. We extend the results of Ref.[2] to the case of multiply-connected domains and identify the set of growth processes with a universal Whitham hierarchy of integrable equations. The latter unveils the mathematical structure of the growth and set a place for growth models in the realm of soliton theory.

Laplacian growth, also known as the Hele-Shaw problem, refers to dynamics of a moving front (an interface) between two distinct phases driven by a harmonic scalar field. This field is a potential for the growth velocity field. The Laplacian growth problem appears in different physical and mathematical contexts and has a number of important practical applications. The most known ones are filtration processes in porous media, viscous fingering in the HeleShaw cell, electrodeposition and solidification in undercooled liquids. A comprehensive list of relevant papers published prior to 1998 can be found in [3].

The most interesting and the most studied dynamics occurs in two-dimensional spatial geometry. To be definite, we shall speak about an interface between two incompressible fluids with very different viscosities on the plane. In practice the $2 \mathrm{D}$ geometry is realized in a Hele-Shaw cell - a narrow gap between two parallel plates (Fig. 1). In this version, the problem is also known as the Saffman-Taylor problem or viscous fingering. For a review, see [4]. Importance of studies of the Laplacian growth with more than one nonviscous droplet speaks for itself. When rates of flow are considerable, then, because of fingering instability, new droplets are pinched off and change their shapes, so the whole dynamics considerably changes in comparison with a single bubble dynamics. (See, e.g., experimental works [5].)

To be more precise, consider the case when there are several disconnected domains in the Hele-Shaw cell occupied by a fluid with low viscosity (water). We call them water droplets. Their exterior, which is in general a multiply-connected domain, is occupied by a viscous fluid (oil). All components of the oil/water interface are assumed to be smooth curves. Oil is sucked out with fixed rates $Q_{j}$ through sinks placed at some finite points $a_{j}$ or at infinity (edges of the Hele-Shaw cell). Water is injected into each water droplet with rates $q_{\alpha}$, some of which may be negative or equal to zero. Hele-Shaw flows in general setting, including the multiply-connected case, were discussed in [6]-[11].

Viscous flows are governed by gradient of the pressure field in the fluids. In the oil domain, the local velocity $\vec{V}=\left(V_{x}, V_{y}\right)$ of the fluid is proportional to the gradient of pressure $p=p(x, y)$ (Darcy's law): $\vec{V}=-\kappa \nabla p$, where $\kappa$ is called the filtration coefficient. In what follows, we choose units in such a way that $\kappa=\frac{1}{4}$. In particular, the Darcy law holds on the outer side of the interface thus governing its dynamics:

$$
V_{n}=-\frac{1}{4} \partial_{n} p
$$




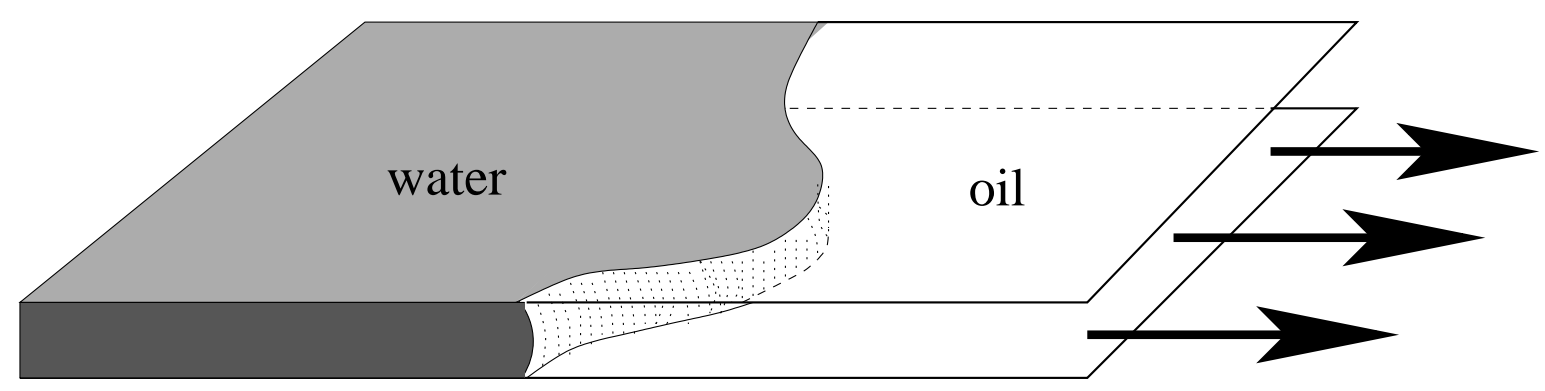

Figure 1: The Hele-Shaw cell (arrows show direction of the flow forced by a pump).

Here $\partial_{n}$ is the normal derivative.

This simple dynamics results to complicated unstable patterns often growing beyond control. The most recent experimentally produced pattern can be seen in Ref. [5].

In this paper we discuss only the idealized problem, namely that surface tension equals zero. We call it the idealized Laplacian growth (ILG). Zero surface tension means that pressure does not jump across the boundary. Assuming that viscosity of water is small enough comparing to the viscosity of oil, pressure is constant inside each water droplet. However, the values of pressure may be different in different droplets and may also depend on time. Let $p_{\alpha}$ be pressure in the $\alpha$-th droplet, then zero surface tension implies that $p=p_{\alpha}$ on the outer side of the interface as well.

Since fluids are incompressible $(\nabla \vec{V}=0)$ the Darcy law implies that the pressure field $p$ is a harmonic function in the exterior (oil) domain except at the points where the oil pumps are located. In the case of zero surface tension, pressure is a solution of the time-dependent boundary problem for the Laplace equation with $p=p_{\alpha}$ on the boundary components. The interface moves according to the Darcy law (1), so that the boundary problem changes with time. Note that the problem is non-local since the gradient of pressure around boundary depends on the shape of the domain as a whole.

When the interface bounds a simply-connected domain, an effective tool for dealing with the Laplacian growth is the time dependent conformal mapping technique (see e.g. [4]). Passing to the complex coordinates $z=x+i y, \bar{z}=x-i y$ on the physical plane, one may describe dynamics in terms of a moving conformal map from a simple reference domain, say the unit disk in a "mathematical plane", onto a growing domain in the physical plane.

If the interface has several disconnected components the conformal map approach meets fundamental difficulties. Uniformizing maps of multiply-connected domains are essentially more complicated mainly because there are no simple reference domains and, moreover, any possible reference domain depends on the growing domain itself.

Instead, given growing domains, one may construct an algebraic curve over complex numbers, or a Riemann surface, endowed with some additional structures. Then the Darcy law translates the growth to an evolution of the algebraic curve, thus providing a remarkable family of flows on the (extended) moduli space of Riemann surfaces.

This is the meeting point of the growth processes and soliton theory. We show that the evolution of the Riemann surface built upon the moving interface in the Hele-Shaw cell is 
governed by the universal Whitham hierarchy of soliton theory. It generalizes the dispersionless 2D Toda hierarchy which was shown in Ref. [2] to describe the moving boundary problems in the simply-connected case. The Whitham equations have been originally introduced to describe slow modulations of periodic solutions to differential equations [12]. Their relation to the algebraic geometry, using example of the KdV equation, was found in [13]. In general setting, the universal Whitham hierarchy was introduced in [14, 15, 16].

The mathematical equivalence between the Laplacian growth with zero surface tension and the Whitham equations, established in this work, allows one to treat the former as a singular limit of dispersive waves obeying soliton equations. Such a singular limit may often lead to solutions which develop singularities within finite time.

In the context of the Laplacian growth, the singularities show up as cusps generated by an initially smooth interface [19], after which the idealized description no longer makes any physical sense. This feature signifies that the very problem with zero surface tension is ill-posed.

A similar difficulty has been known to exist for the Whitham equations (see, e.g., [17]). Some of their solutions, being initially regular, sooner or later become singular and so can not be extended to all times. The Korteweg de Vries equation with zero dispersion (the Hopf equation) is the most familiar example. Here any smooth decreasing Cauchy data evolve into a "shock wave" with an overturned front which is physically meaningless (a "gradient catastrophe"). This simply means that the dispersionless approximation does not work in a vicinity of the catastrophe. Similarly, the zero surface tension assumption is not valid in a vicinity of the cusp formation.

These singularities are in fact artificial and can be successfully resolved by methods developed in the theory of slow modulations of exact periodic solutions to soliton equations $[1,18]$. In subsequent works we hope to apply these methods to the Laplacian growth using the proven below equivalence between the two disciplines.

\section{Linearization of the ILG dynamics}

Remarkably, the ILG dynamics, initially formulated as a non-local and highly nonlinear problem, admits an exact linearization in the space of harmonic moments of the viscous domain. By linearization we mean here a change of variables which converts the nontrivial LG dynamics into a simple linear one. A familiar (but rather loose) analogy is passing to action-angle variables in classical mechanics or the inverse scattering transform in the soliton theory.

Our starting point is the fact that the ILG is a simple linear shift in the space of harmonic moments of the growing domain. This statement goes back to seminal Richardson's paper [6]. In that paper, it was shown that if there is the only sink at infinity, all moments are conserved except the moment of constant function (the area of the droplet) which changes linearly with time. In fact, it is absolutely clear that for incompressible fluids and fixed pumping rates areas of the droplets, if change at all, always do this linearly with time. For arbitrary location of the sink, and also for several sinks at different points, a simple extension 
of this result states that in general all moments change linearly with time (with different coefficients which may be zero).

In the case of several water droplets, the set of harmonic moments should be supplemented by a finite number of extra parameters, one for each extra droplet, which are basically moments of harmonic functions with multivalued analytic parts. This set of variables is enough to characterize the geometry of the growing domain. Alternatively, the new parameters may be areas of the water droplets. Depending on which type of external physical conditions in the water droplets is realized (fixed pressure differences or fixed pumping rates), the ILG dynamics becomes linear either in the former or in the latter variables.

Most of the material of Secs. 2.1 and 2.2 is spread through the literature (see, e.g., $[6,7,8,9]$ and Chapter 5 of the book [11]). To make our exposition self-contained, we review them from a unifying point of view.

\subsection{The time-dependent boundary problem}

Consider an ILG process with the point-like oil pumps with powers $Q_{j}$ at some points $a_{j}$ located far enough from the moving interface. Mathematically this means

$$
-\oint_{\mathrm{c}_{j}} V_{n} d s=\pi Q_{j}
$$

where $c_{j}$ is a small contour encircling the point $a_{j}$, $d s$ stands for the differential of the arc length, $V_{n}$ is the component of the fluid velocity normal to the contour, with the normal vector pointing outside the circle $\mathrm{c}_{j}$. Both $Q_{j}$ and $a_{j}$ are assumed to be time-independent. Oil may be also sucked at infinity. Physically this means, for example, that oil is removed from the edge of a large Hele-Shaw cell. Mathematically one puts one of $a_{j}$ equal to infinity and defines the pumping rate at infinity, $Q_{\infty}$, as

$$
-\oint_{c_{\infty}} V_{n} d s=\pi Q_{\infty}
$$

Here $c_{\infty}$ is a big contour encircling the whole system of water droplets and all the point-like pumps, if any. The oil pumping rates are assumed to be positive when oil is sucked and negative if it is injected into the Hele-Shaw cell.

One can also consider extended sources or sinks of oil, for instance, continuously distributed along lines, like in Ref. [9]. To avoid irrelevant technical complications, we consider point-like oil pumps only, giving brief remarks on the more general case when necessary.

Let $D_{\text {oil }}$ be the region of the plane occupied by oil (an infinite domain containing infinity), and $\mathrm{D}$ be the region occupied by water. We assume that there are $g+1$ water droplets in the Hele-Shaw cell, which are compact domains bounded by smooth non-intersecting curves. Let $\mathrm{D}_{\alpha}$ be the $\alpha$-th water droplet, $\alpha=0,1, \ldots, g$, so that $\mathrm{D}$ is their union (Fig. 2). It is convenient to think of the 0-th droplet as of the "main" one, having in mind that it is this droplet that remains in the system in the simply-connected case. Let us assume, just for a notational convenience, that the origin is in the zero droplet, $0 \in \mathrm{D}_{0}$. 


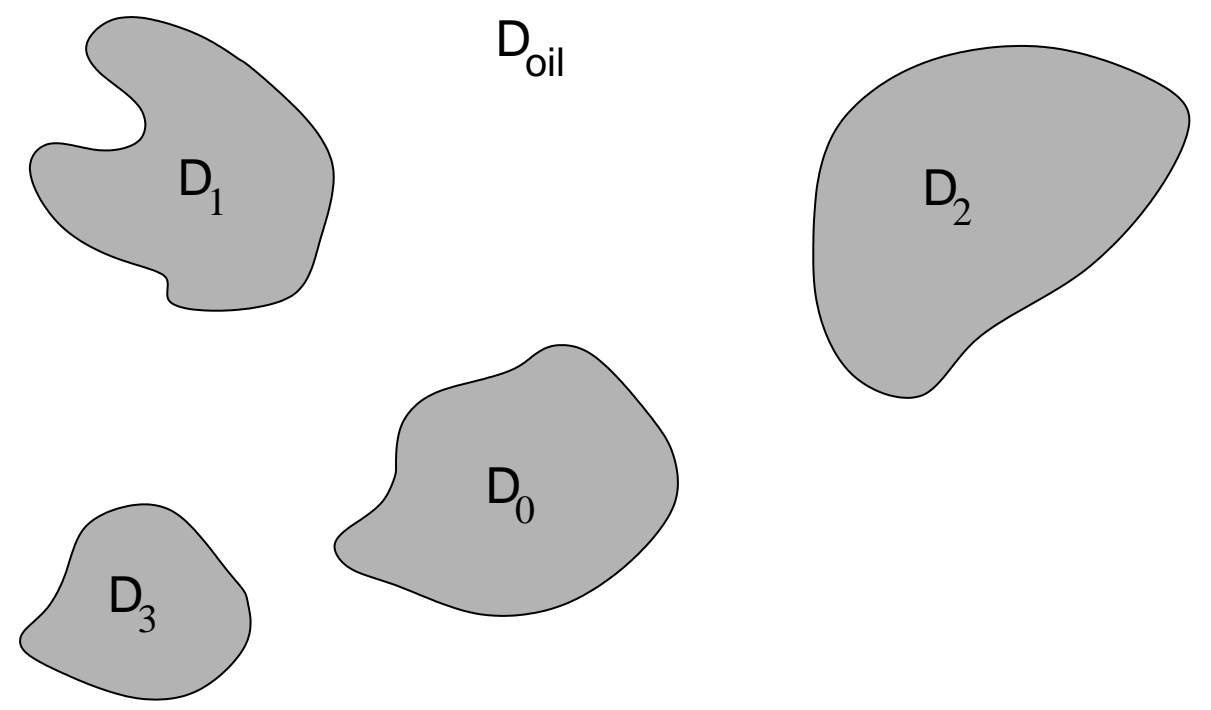

Figure 2: Water droplets $\mathrm{D}_{\alpha}(g=3)$.

The incompressibility implies that the oil pumps are able to work only if there are some sources of water which supply water to at least one of the droplets. We call them water pumps and introduce the pumping rate $q_{\alpha}$ of water in the $\alpha$-th droplet:

$$
\oint_{\partial \mathrm{D}_{\alpha}} V_{n} d s=\pi q_{\alpha}
$$

Here the normal vector points outside $\mathrm{D}_{\alpha}$. The pumping rate $q_{\alpha}$ is assumed to be positive when water is injected into the $\alpha$-droplet. Obviously, the oil and water pumping rates are constrained by the relation

$$
Q_{\infty}+\sum_{j} Q_{j}=\sum_{\alpha} q_{\alpha}
$$

The pressure field in oil obeys the equation

$$
\Delta p(z)=4 \pi \sum_{j} Q_{j} \delta^{(2)}\left(z-a_{j}\right),
$$

where $\delta^{(2)}(z)$ is the two-dimensional delta-function and $\Delta=\partial_{x}^{2}+\partial_{y}^{2}=4 \partial_{z} \partial_{\bar{z}}$ is the Laplace operator on the plane. In other words, $p=p(z)$ is a harmonic function in $\mathrm{D}_{\text {oil }}$ with the asymptote

$$
p(z)=Q_{j} \log \left|z-a_{j}\right|^{2}+\ldots \quad \text { in the vicinity of } a_{j}
$$

Taking into account the Darcy law, this agrees with the definition of the $Q_{j}(2)$. On the $\alpha$-th component of the interface the pressure field takes a constant value $p_{\alpha}$ (which may depend on time). Without loss of generality, we set $p_{0}=0$.

To determine velocity of the interface, one should solve a time-dependent Dirichlet-like boundary problem. It has a unique solution, and so pressure in $\mathrm{D}_{\text {oil }}$ is uniquely determined as soon as one specifies pressures $p_{\alpha}$ in water droplets. This means that with given $p_{\alpha}$ 's there is no freedom in water pumping. Indeed, the pumping rates in all water droplets are to be 
determined from (3), which states that $q_{\alpha}=-\frac{1}{4 \pi} \oint_{\partial \mathrm{D}_{\alpha}} \partial_{n} p d s$. If $p_{\alpha}$ 's are maintained constant with time, $q_{\alpha}$ 's are in general certain complicated functions of time. Alternatively, one may control the pumping rates $q_{\alpha}$ keeping them constant, then pressures in water droplets are uniquely determined by the dynamical equations and in general exhibit a nontrivial time dependence.

So the physical problem is not yet defined by the local growth law alone. One should add some physical conditions in water droplets. We distinguish two cases:

(I) Fixed pressure differences $p_{\alpha}: \dot{p}_{\alpha}=0$, then $q_{\alpha}=q_{\alpha}(t)$ are to be determined;

(II) Fixed pumping rates $q_{\alpha}: \dot{q}_{\alpha}=0$, then $p_{\alpha}=p_{\alpha}(t)$ are to be determined

Here the dot means the time derivative. Various "mixed" conditions (say, when we fix some of $p_{\alpha}$ 's and some of $q_{\alpha}$ 's or linear combinations thereof) are not meaningless from the mathematical point of view but look somewhat artificial in the physical context.

For an abuse of quantum-mechanical terminology, one may refer to the cases (I) and (II) as the LG problem in $p$-representation and $q$-representation, respectively.

\subsection{Integral transforms of the oil domain}

The Cauchy transform. Here we closely follow Richardson's paper [8]. Let us consider the Cauchy transform of the oil domain:

$$
h(z)=\frac{1}{\pi} \int_{\mathrm{D}_{\mathrm{oil}}} \frac{d^{2} \zeta}{z-\zeta}
$$

(A cut-off at some radius $R$ is implied, at large $R$ the integral does not depend on the cut-off which may be safely moved to infinity.) This function is continuous across all boundaries and analytic for $z$ in each hole $D_{\alpha}$ while in $D_{\text {oil }}$ the function $h(z)-\bar{z}$ is analytic. So we can write

$$
h(z)=\left\{\begin{array}{l}
h_{\alpha}^{+}(z) \quad \text { for } z \text { in } \mathrm{D}_{\alpha} \\
\bar{z}+h^{-}(z) \text { for } z \text { in } \mathrm{D}_{\text {oil }}
\end{array}\right.
$$

where $h_{\alpha}^{+}(z)$ is an analytic function in $\mathrm{D}_{\alpha}$ and $h^{-}(z)$ is analytic for $z$ in $\mathrm{D}_{\text {oil }}$. One may analytically continue the functions $h_{\alpha}^{+}, h^{-}$outside the regions where they are defined by the Cauchy transform. In general, all $g+1$ functions $h_{\alpha}^{+}(z)$ obtained in this way are expected to be different.

The contour integral representation of these functions reads:

$$
\frac{1}{2 \pi i} \oint_{\partial \mathrm{D}} \frac{\bar{\zeta} d \zeta}{\zeta-z}=\left\{\begin{array}{l}
h_{\alpha}^{+}(z) \text { for } z \text { in } \mathrm{D}_{\alpha} \\
h^{-}(z) \text { for } z \text { in } \mathrm{D}_{\text {oil }}
\end{array}\right.
$$

Note that $h_{\alpha}^{+}(z)-h^{-}(z)=\bar{z}$ on the $\alpha$-th boundary curve. 
The time derivative $\dot{h}(z)=\partial_{t} h(z ; t)$, is found straightforwardly using the integral representation (5) and the Darcy law:

$$
\begin{gathered}
\dot{h}(z)=-\frac{1}{\pi} \oint_{\partial \mathrm{D}_{\mathrm{oil}}(t)} \frac{1}{z-\zeta} V_{n} d s=\frac{1}{4 \pi} \oint_{\partial \mathrm{D}_{\mathrm{oil}}(t)} \frac{1}{z-\zeta} \partial_{n} p(\zeta) d s= \\
=\frac{1}{4 \pi} \oint_{\partial \mathrm{D}_{\mathrm{oil}}(t)}\left(\frac{1}{z-\zeta} \partial_{n} p(\zeta)-p(\zeta) \partial_{n} \frac{1}{z-\zeta}\right) d s+\frac{1}{4 \pi} \sum_{\alpha=1}^{g} p_{\alpha} \oint_{\partial \mathrm{D}_{\alpha}(t)} \partial_{n} \frac{1}{z-\zeta} d s
\end{gathered}
$$

where we subtracted and added the integral of $p \partial_{n} \frac{1}{z-\zeta}$ over the whole boundary, and used the fact that $p$ is constant along any component of the boundary. It is easy to see that irrespectively of whether the point $z$ is inside or outside $D_{\alpha}$, all contour integrals in the second term vanish. The first term can be transformed using the Green theorem:

$$
\oint_{\partial \mathrm{D}_{\mathrm{oil}}(t)}\left(\frac{1}{z-\zeta} \partial_{n} p-p \partial_{n} \frac{1}{z-\zeta}\right) d s=-\int_{\mathrm{D}_{\mathrm{oil}}(t)}\left(\frac{1}{z-\zeta} \Delta p(\zeta)-p(\zeta) \Delta \frac{1}{z-\zeta}\right) d^{2} \zeta
$$

The Laplacian of $p(\zeta)$ is given by (4). If $z$ is inside a water droplet, the function $\frac{1}{z-\zeta}$ (regarded as a function of $\zeta$ ) is harmonic for all $\zeta$ in $D_{\text {oil }}$ and the second term vanishes. If $z$ is in $D_{\text {oil }}$,

$$
\int_{\mathrm{D}_{\mathrm{oil}}(t)} p(\zeta) \Delta_{\zeta}\left(\frac{1}{z-\zeta}\right) d^{2} \zeta=4 \pi \partial_{z} p(z)
$$

Finally, we get:

$$
\begin{gathered}
\dot{h}_{\alpha}^{+}(z)=\sum_{j} \frac{Q_{j}}{a_{j}-z} \quad \text { for } z \text { in } \mathrm{D}_{\alpha} \\
\dot{h}^{-}(z)=\sum_{j} \frac{Q_{j}}{a_{j}-z}+\partial_{z} p(z) \quad \text { for } z \text { in } \mathrm{D}_{\text {oil }}
\end{gathered}
$$

In the integrated form,

$$
h_{\alpha}^{+}(z ; t)=h_{\alpha}^{+}(z ; 0)+t \sum_{j} \frac{Q_{j}}{a_{j}-z}
$$

We see that the increment of the $h_{\alpha}^{+}$is a rational function, and, moreover, it is the same for all $\alpha=0,1, \ldots, g$. We also see that this function is entirely determined by the output powers and locations of oil pumps, no matter what conditions we impose on the pressures and pumping rates in water droplets.

For a linearly distributed oil source, $p(z)$ is a potential of a simple layer. Then one finds that the functions $\dot{h}_{\alpha}^{+}$, though no longer rational, are still analytic continuations of a single analytic function unless the support of the simple layer forms a noncontractable cycle encircling at least one of the water droplets.

A remarkable property of the Cauchy transform is its linear dependence on time $t$ (provided the parameters of the oil pumps are time-independent). This is the key point that allows one to linearize the ILG dynamics. 
The Coulomb potential. Along with the Cauchy transform of the domain $\mathrm{D}_{\text {oil }}$ it is useful to consider the potential generated by fictitious 2D Coulomb charges uniformly distributed in $D_{\text {oil }}$ :

$$
\phi(z)=\frac{1}{\pi} \int_{\mathrm{D}_{\mathrm{oil}}} \log \left|1-\frac{z}{\zeta}\right|^{2} d^{2} \zeta
$$

(The same cut-off as in the Cauchy transform is implied.) Clearly, this function is harmonic in each water droplet and its $z$-derivative coincides with the $h(z): \partial_{z} \phi(z)=h(z)$. Repeating the above calculation for $\phi(z)$ we get (for $z$ in water droplets):

$$
\dot{\phi}(z)=-\frac{1}{4 \pi} \int_{\mathrm{D}_{\text {oil }}} \log \left|1-\frac{z}{\zeta}\right|^{2} \Delta p(\zeta) d^{2} \zeta+\frac{1}{4 \pi} \sum_{\alpha=1}^{g} p_{\alpha} \oint_{\partial \mathrm{D}_{\alpha}} \partial_{n} \log \left|1-\frac{z}{\zeta}\right|^{2} d s
$$

This yields:

$$
\dot{\phi}(z)=p_{\alpha}-\sum_{j} Q_{j} \log \left|1-\frac{z}{a_{j}}\right|^{2} \quad \text { for } z \text { in } \mathrm{D}_{\alpha}
$$

The Laplacian growth equation. For completeness, let us demonstrate that the Laplacian growth equation, usually derived, in the simply-connected case, using the time dependent conformal map technique, follows from the time derivative of the Cauchy transform.

To this end, we calculate the difference of the boundary values $\dot{h}^{ \pm}$in two ways. On the one hand, it is obtained by subtracting (8) and (9):

$$
\dot{h}^{+}(z)-\dot{h}^{-}(z)=-\partial_{z} p(z), \quad \text { for } z \text { on any boundary contour }
$$

On the other hand,

$$
\partial_{t} h^{ \pm}(z)=\frac{1}{2 \pi i} \partial_{t}\left(\oint_{\partial \mathrm{D}} \frac{\bar{\zeta} d \zeta}{\zeta-z}\right)
$$

can be found directly using a parametrization of the family of contours $z=z(\sigma, t)$, where $\sigma$ is a parameter along the contour. For each component of the boundary we have:

$$
\begin{gathered}
\partial_{t}\left(\oint \frac{\bar{z} d z}{z-a}\right)=\int\left(\frac{\overline{z_{t}} z_{\sigma}+\bar{z} z_{\sigma t}}{z-a}-\frac{\bar{z} z_{\sigma} z_{t}}{(z-a)^{2}}\right) d \sigma= \\
\text { (integrating by parts) }=\oint\left(\frac{\overline{z_{t}} z_{\sigma}-\overline{z_{\sigma}} z_{t}}{z-a}\right) \frac{d z}{z_{\sigma}}
\end{gathered}
$$

The jump of the boundary values of the analytic function defined by the latter Cauchy integral is equal to $\overline{z_{t}}-\overline{z_{\sigma}} z_{t}\left(z_{\sigma}\right)^{-1}$. Combining this with (13), we obtain the relation for differentials along the boundary curves,

$$
\partial_{t} \overline{z(\sigma, t)} d z-\partial_{t} z(\sigma, t) d \bar{z}=-\partial_{z} p(z) d z
$$

valid for any parametrization of the contours. In a simply-connected case with an oil sink at infinity $p(z)=-2 Q_{\infty} \log |w(z)|$, where $w(z)$ is a conformal map of the oil domain to the exterior of the unit disk. Choosing $\sigma=-i \log w(z)$ Eq.(14) becomes the celebrated Laplacian growth equation

$$
\overline{z_{t}} z_{\sigma}-z_{t} \overline{z_{\sigma}}=i Q_{\infty}
$$

It was first derived in [20]. 


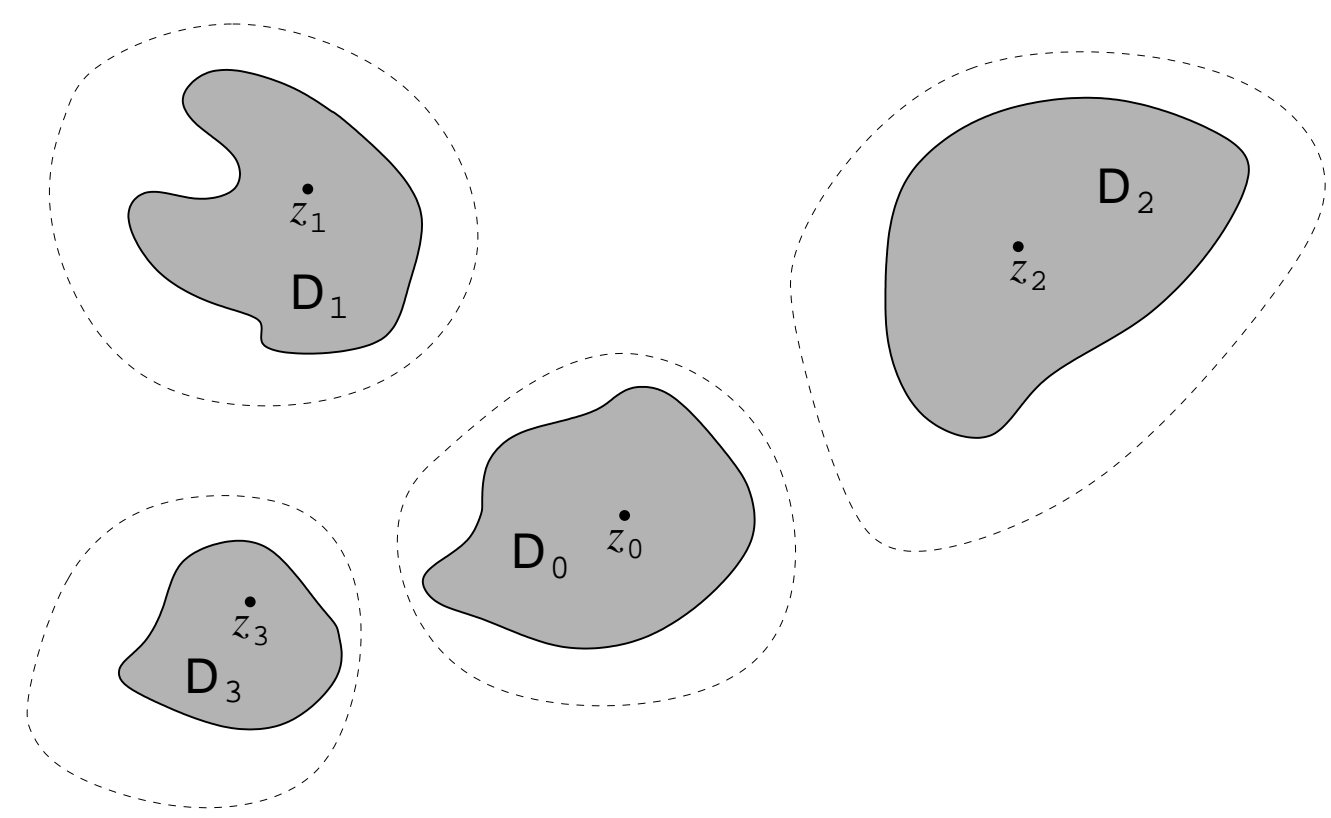

Figure 3: The domain $\tilde{\mathrm{D}}_{\text {oil }}$ is the exterior of the regions bounded by the dashed lines.

\subsection{Dual systems of local coordinates in the space of multiply- connected domains}

Our next goal is to introduce special local coordinates in the space of multiply-connected domains, which evolve linearly in time. The time evolution of the Cauchy transform suggests that such coordinates are basically harmonic moments of the oil domain.

The proper basis of harmonic functions. Let us consider a time-independent domain $\tilde{D}_{\text {oil }} \subset D_{\text {oil }}$ with the same connectivity as $D_{\text {oil }}$, as shown in Fig. 3 , and define a proper basis of harmonic functions in $\tilde{D}_{\text {oil }}$. A basis is said to be proper if any harmonic function in $D_{\text {oil }}$ is representable as a linear combination (possibly infinite) of the basis functions such that it converges everywhere in $\tilde{D}_{\text {oil }}$. In the case of a single water droplet, the basis consisting of functions $z^{-n}$ and their conjugates is clearly a proper one. However, this basis is no longer proper on the plane with more than one hole. Indeed, in this case one has to incorporate functions with singularities in any hole, not only in $\mathrm{D}_{0}$, otherwise the series converges only in some simply-connected neighborhood of infinity.

To construct a proper basis, we need some auxiliary data. Inside each water droplet, let us fix a point, $z_{\alpha} \in \mathrm{D}_{\alpha}$, which does not move with time (Fig. 3). These points may be thought of as locations of point-like water pumps. Without any loss of generality, we set $z_{0}=0$.

Consider the following single-valued analytic functions in $\mathrm{D}_{\mathrm{oil}}$ :

$$
\psi_{k}(z)=\prod_{\gamma=0}^{k-1} \frac{1}{z-z_{\gamma}}, \quad k \geq 0,
$$


where the running index $\gamma$ is understood modulo $g+1$, i.e., it is implied that $z_{\gamma+(g+1) m} \equiv z_{\gamma}$. At $k=0$ we set $\psi_{0}=1$. In a more explicit form, we write

$$
\psi_{(g+1) m+\alpha}=r^{-m}(z) \prod_{\beta=0}^{\alpha-1}\left(z-z_{\beta}\right)^{-1}
$$

where $m \geq 0, \alpha=0,1, \ldots, g$ (when $\alpha=0$ the product is set to be 1 ) and

$$
r(z)=\prod_{\beta=0}^{g}\left(z-z_{\beta}\right)
$$

These functions constitute a proper basis of single-valued analytic functions. (As is seen from (16), the domain $\tilde{\mathrm{D}}_{\text {oil }}$ can be choosen to be $|r(z)|>C$ for some positive $C$.) This basis is a simplified version of the special Laurent-Fourier type basis on Riemann surfaces constructed in [21]. In the case of the single hole $\mathrm{D}_{0}$ with $z_{0}=0$ it coincides with the standard one: $\psi_{k}=z^{-k}$.

Any harmonic function is the real part of an analytic function. However, in the multiplyconnected case these analytic functions are not necessarily single-valued (only their real parts have to be single-valued). The full basis of harmonic functions is, therefore: $\psi_{k}(z), \overline{\psi_{k}(z)}$, $k \geq 1, \psi_{0}=1$, and $g$ functions of the form

$$
\ell_{\alpha}(z)=\log \left|1-\frac{z_{\alpha}}{z}\right|^{2}, \quad \alpha=1, \ldots, g
$$

which have multivalued analytic part.

An important feature of the basis $\psi_{k}$ is the existence of the "dual" basis of differentials, $d \chi_{k}(z)$, such that

$$
\frac{1}{2 \pi i} \oint_{\partial \mathrm{D}} \psi_{k} d \chi_{n}=\delta_{k n}
$$

Explicitly, they are given by

$$
d \chi_{k}(z)=\frac{d z}{\left(z-z_{k-1}\right) \psi_{k}(z)}=d z \prod_{\gamma=0}^{k-2}\left(z-z_{\gamma}\right)
$$

where $z_{k}$ is again understood as $z_{\alpha}$ where $k=(g+1) m+\alpha$ with non-negative integer $m$ and $0 \leq \alpha \leq g$. It is clear that $\chi_{k}^{\prime}(z)=d \chi_{k} / d z$, for $k \geq 1$, are polynomials of degree $k-1$.

One can easily check that the following expansion of the Cauchy kernel holds true:

$$
\frac{1}{\zeta-z}=\sum_{k=1}^{\infty} \psi_{k}(\zeta) \chi_{k}^{\prime}(z)
$$

and the series converges if $|r(z)|<|r(\zeta)|$. In particular, the series certainly converges if $\zeta$ is in $D_{\text {oil }}$ while $z$ is close enough to any one of the points $z_{\alpha}$. A similar expansion holds for the logarithmic kernel:

$$
\log (\zeta-z)=\log \left(\zeta-z_{\alpha}\right)-\sum_{k=1}^{\infty} \psi_{k}(\zeta) \int_{z_{\alpha}}^{z} d \chi_{k} \quad \text { for } \zeta \text { in } \mathrm{D}_{\text {oil }} \text { and } z \text { in } \mathrm{D}_{\alpha}
$$

with the same condition of convergence. 
The harmonic moments. Using the expansion of the Cauchy kernel (21), let us expand the function $h^{+}(z)$ in a series, which converges at least for $z$ close enough to any one of the points $z_{\alpha}$. We obtain:

$$
h^{+}(z)=\sum_{k \geq 1} k T_{k} \chi_{k}^{\prime}(z)
$$

where

$$
T_{k}=-\frac{1}{\pi k} \int_{D_{\mathrm{oil}}} \psi_{k}(\zeta) d^{2} \zeta
$$

are normalized harmonic moments of the domain $\mathrm{D}_{\text {oil }}$. (In the case of the single water droplet $\mathrm{D}_{0}$ they have the familiar form $T_{k}=-\frac{1}{\pi k} \int_{\mathrm{D}_{\mathrm{oil}}} \zeta^{-k} d^{2} \zeta$.) Their time derivatives are read from $(8)$ :

$$
\dot{T}_{k}=\frac{1}{k} \sum_{j} Q_{j} \psi_{k}\left(a_{j}\right)
$$

In case of the only sink at infinity the r.h.s. vanishes [6]. The moment $T_{0}$ is the area of $\mathrm{D}_{\text {oil }}$. It is infinite, but as long as we need only its change, we may equivalently consider the area of the complimentary domain

$$
T_{0}=\frac{1}{\pi} \sum_{\alpha=0}^{g} \text { Area }\left(\mathrm{D}_{\alpha}\right)
$$

Evidently, $\dot{T}_{0}$ is equal to the total power of oil pumps: $\dot{T}_{0}=Q_{\infty}+\sum_{j} Q_{j}$.

To characterize the geometry of a multiply-connected domain with $g+1$ boundary components one needs $g$ extra parameters. There are different possibilities to choose them. One of possible choices is as follows. Using the expansion of the logarithmic kernel (22), or integrating the expansion of the $h(z)$, we can represent the Coulomb potential function in the form

$$
\phi(z)=\Phi_{\alpha}+2 \operatorname{Re}\left(\sum_{k \geq 1} k T_{k} \int_{z_{\alpha}}^{z} d \chi_{k}\right) \quad \text { for } z \text { in } \mathrm{D}_{\alpha}
$$

where $\Phi_{\alpha}=\phi\left(z_{\alpha}\right)$ are integration constants. Since $\phi(0)=0$, we set $\Phi_{0}=0$. The other integration constants, $\Phi_{1}, \ldots, \Phi_{g}$, may serve as the additional independent parameters. Clearly,

$$
\Phi_{\alpha}=\frac{1}{\pi} \int_{\mathrm{D}_{\mathrm{oi} 1}} \log \left|1-\frac{z_{\alpha}}{\zeta}\right|^{2} d^{2} \zeta
$$

are harmonic moments of the $D_{\text {oil }}$ with respect to the functions $\ell_{\alpha}(z)(18)$. These moments are always real. The time derivative $\dot{\Phi}_{\alpha}=\dot{\phi}\left(z_{\alpha}\right)$ is given by (12):

$$
\dot{\Phi}_{\alpha}=p_{\alpha}-\sum_{j} Q_{j} \log \left|1-\frac{z_{\alpha}}{a_{j}}\right|^{2}=p_{\alpha}-\sum_{j} Q_{j} \ell_{\alpha}\left(a_{j}\right)
$$

There is an alternative choice of the $g$ extra parameters which is "dual" to the choice above. Let

$$
S_{\alpha}=\frac{\operatorname{Area}\left(\mathrm{D}_{\alpha}\right)}{\pi}
$$


be areas of the water droplets (divided by $\pi$ ), then

$$
\dot{S}_{\alpha}=q_{\alpha}
$$

by the definition of $q_{\alpha}$. Clearly, $\sum_{\alpha=0}^{g} S_{\alpha}=T_{0}$, so $S_{1}, \ldots, S_{g}$ can be taken as independent parameters.

Local coordinates in the space of multiply-connected domains. The basic fact from the theory of deformations of planar domains (which we adopt without proof in this paper) is that the parameters $T_{k}, \Phi_{\alpha}$ or $T_{k}, S_{\alpha}$ can serve as local coordinates in the space of planar multiply-connected domains. This means that any deformation which preserves these parameters is trivial and any vector field in the space of these parameters generates a welldefined deformation of the initial domain (see [22] for details). Recovery of the domain having these parameters constitutes the classical inverse potential problem [23] for the multiplyconnected case. The fact that the coordinates introduced above are good ones amounts to local existence and uniqueness of such a domain. An effective reconstruction, however, is not generally feasible and even specific examples are challenging and merit separate attention.

The formulas for time derivatives of $T_{k}, \Phi_{\alpha}$ and $S_{\alpha}$ tell us that any ILG flow can be represented, in these coordinates, as a vector field with constant coefficients.

To summarize, we have introduced two systems of local coordinates in the space of multiply-connected domains:

- $T \Phi$-coordinates: the harmonic moments $T_{0}, T_{1}, T_{2}, \ldots$ (and their complex conjugates) and $\Phi_{1}, \Phi_{2}, \ldots, \Phi_{g}$

- $T S$-coordinates: the harmonic moments $T_{0}, T_{1}, T_{2}, \ldots$ (and their complex conjugates) and $S_{1}, S_{2}, \ldots, S_{g}$.

Now, from (24), (26) and (28) it is clear that Richardson's result can be reformulated by saying that the ILG dynamics with fixed pressure differences (the $p$-representation) is linearized in the $T \Phi$-coordinates while the ILG dynamics with fixed pumping rates (the $q$ representation) becomes linear in $T S$-coordinates.

\subsection{Elementary growth processes}

A linear time dependence of the local coordinates suggests to treat any general ILG process as a superposition of certain "elementary" processes. It is natural to associate with each elementary flow its own time variable.

In the $p$-representation, the elementary processes are:

(p1) Oil is sucked from a point $a$ with the unit rate $(Q=1)$, with maintaining equal pressures in all droplets $\left(p_{\alpha}=0\right)$ :

$$
\dot{T}_{0}=1, \quad \dot{T}_{k}=\frac{1}{k} \psi_{k}(a), \quad \dot{\Phi}_{\alpha}=-\ell_{\alpha}(a)
$$


With this process we associate the time variable $T^{(a)}$ such that

$$
\frac{\partial}{\partial T^{(a)}}=\frac{\partial}{\partial T_{0}}+\sum_{k \geq 1} \frac{1}{k}\left(\psi_{k}(a) \frac{\partial}{\partial T_{k}}+\overline{\psi_{k}(a)} \frac{\partial}{\partial \bar{T}_{k}}\right)-\sum_{\alpha} \ell_{\alpha}(a) \frac{\partial}{\partial \Phi_{\alpha}}
$$

(the values of the coefficients follow from (24), (26)). Note that $T^{(a)}$ is the amount of oil sucked out from the point $a$ during the process.

(p2) Water is redistributed between the droplets by applying the unit pressure difference between the $\alpha$-th and the 0 -th droplets: $p_{\beta}=\delta_{\alpha \beta}$, with no pumps in oil:

$$
\dot{T}_{0}=\dot{T}_{k}=0, \quad \dot{\Phi}_{\beta}=\delta_{\alpha \beta}
$$

With this process we associate the time variable $T^{(\alpha)}$ such that

$$
\frac{\partial}{\partial T^{(\alpha)}}=\frac{\partial}{\partial \Phi_{\alpha}}
$$

It is the amount of water injected into the 0-th water droplet during the process.

In the $q$-representation, the elementary processes are:

(q1) Oil is sucked from a point $a$ with the unit rate $(Q=1)$, with water being added to the 0-th droplet only $\left(q_{0}=1\right)$ :

$$
\dot{T}_{0}=1, \quad \dot{T}_{k}=\frac{1}{k} \psi_{k}(a), \quad \dot{S}_{\beta}=0
$$

In this case the vector field $\partial / \partial T^{(a)}$ is represented as

$$
\frac{\partial}{\partial T^{(a)}}=\frac{\partial}{\partial T_{0}}+\sum_{k \geq 1} \frac{1}{k}\left(\psi_{k}(a) \frac{\partial}{\partial T_{k}}+\overline{\psi_{k}(a)} \frac{\partial}{\partial \bar{T}_{k}}\right)
$$

(q2) Water is sucked from the $\alpha$-th droplet and injected into the 0-th one with the unit rate: $q_{\beta}=-\delta_{\alpha \beta}$, with no pumps in oil:

$$
\begin{gathered}
\dot{T}_{0}=\dot{T}_{k}=0, \quad \dot{S}_{\beta}=-\delta_{\alpha \beta} \\
\frac{\partial}{\partial T^{(\alpha)}}=-\frac{\partial}{\partial S_{\alpha}}
\end{gathered}
$$

In (29), (31) $\partial / \partial T^{(a)}$ is to be understood not as a partial derivative but as a vector field in the space of $g+1$ contours. By construction, it is an invariant vector field, i.e., it does not depend on the particular basis of harmonic functions and corresponding local coordinates. The same is true for $\partial / \partial T^{(\alpha)}$. For a general process we have:

$$
\frac{\partial}{\partial t}=Q_{\infty} \frac{\partial}{\partial T_{0}}+\sum_{j} Q_{j} \frac{\partial}{\partial T^{\left(a_{j}\right)}}+\sum_{\alpha} p_{\alpha} \frac{\partial}{\partial \Phi_{\alpha}} \quad(p \text {-representation })
$$




$$
\frac{\partial}{\partial t}=Q_{\infty} \frac{\partial}{\partial T_{0}}+\sum_{j} Q_{j} \frac{\partial}{\partial T^{\left(a_{j}\right)}}+\sum_{\alpha} q_{\alpha} \frac{\partial}{\partial S_{\alpha}} \quad \text { (q-representation) }
$$

The vector fields and relations between them are to be understood as acting on any physical quantity depending on the shape of the growing domain. It is important to stress that the linear superposition works only for processes of the same type (i.e., either of the $p$-type or $q$-type).

At fixed positions of oil sinks ILG spans a finite-dimensional subspace of an infinite dimensional variety of $g+1$ - domains. In this subspace $\partial / \partial T^{(a)}$ and $\partial / \partial T^{(\alpha)}$ act as partial derivatives. For example, fix $N$ points $a_{j}$ and consider the variety of contours which can be obtained from some initial configuration of droplets as a result of an ILG process with oil pumps at the points $a_{j}$. The resulting shape of the droplets is uniquely determined (if no singularity occurs) by total amounts of oil sucked out from each point. This configuration space is $N$-dimensional, and $T^{\left(a_{j}\right)}$ are local coordinates in it. Similarly, one may consider a more general configuration space, where additional parameters are amounts of water injected into each droplet.

\section{Analytic and algebro-geometric objects associated to the ILG}

In this section we describe analytic and algebro-geometric objects [24] which emerge in a description of an evolution of multiply-connected domains.

\subsection{Green function, harmonic measures and period matrix}

Pressure in the oil domain is expressed in terms of the following objects:

- $G\left(z, z^{\prime}\right)$ : The Green function of the Dirichlet boundary problem in $\mathrm{D}_{\text {oil }}$. The function $G\left(z, z^{\prime}\right)$ is symmetric and harmonic everywhere in $\mathrm{D}_{\text {oil }}$ in both arguments except $z=z^{\prime}$ where $G\left(z, z^{\prime}\right)=\log \left|z-z^{\prime}\right|+\ldots$ as $z \rightarrow z^{\prime}$; besides, $G\left(z, z^{\prime}\right)=0$ if any of the variables $z, z^{\prime}$ belongs to the boundary. The Green function obeys the equation $\Delta G\left(z, z^{\prime}\right)=$ $2 \pi \delta^{(2)}\left(z-z^{\prime}\right)$.

- $\omega_{\alpha}(z)$ : The harmonic measure of the $\alpha$-th boundary component. The function $\omega_{\alpha}(z)$ is the harmonic function in $D_{\text {oil }}$ such that it is equal to 1 on $\partial D_{\alpha}$ and vanishes on the other boundary curves. Thus the harmonic measure is the solution to the particular Dirichlet problem. The solution is given by

$$
\omega_{\alpha}(z)=-\frac{1}{2 \pi} \oint_{\partial \mathrm{D}_{\alpha}} \partial_{n} G(z, \zeta) d s, \quad \alpha=0,1, \ldots, g
$$

so the harmonic measure is the period of the differential $\partial_{z} G d z$. Obviously, the sum of the harmonic measures of all boundary components, $\partial \mathrm{D}_{\alpha}$, which we call cycles, is equal to 1 . In what follows we consider the linear independent functions $\omega_{\alpha}(z)$ with $\alpha=1, \ldots, g$. 
- $\Omega_{\alpha \beta}$ : The period matrix. Taking integrals of $\omega_{\alpha}(z)$ over nontrivial cycles, we define

$$
\Omega_{\alpha \beta}=-\frac{1}{2 \pi} \oint_{\partial \mathrm{D}_{\beta}} \partial_{n} \omega_{\alpha}(\zeta) d s, \quad \alpha, \beta=1, \ldots, g
$$

The matrix $\Omega_{\alpha \beta}$ is known to be symmetric, non-degenerate and positively-definite. It is called the period matrix because of its direct relation to periods of holomorphic differentials on the Schottky double of the domain $\mathrm{D}_{\text {oil }}$ (see below).

We also need the following "modified" objects, which are dual, with respect to the choice of the basis of canonical cycles (see Sec. 3.2) to the ones introduced above.

- $\tilde{G}\left(z, z^{\prime}\right)$ : The modified Green function [25] defined by

$$
\tilde{G}\left(z, z^{\prime}\right)=G\left(z, z^{\prime}\right)-\sum_{\alpha, \beta=1}^{g} \omega_{\alpha}(z)\left(\Omega^{-1}\right)_{\alpha \beta} \omega_{\beta}\left(z^{\prime}\right)
$$

This function obeys the same equation $\Delta \tilde{G}\left(z, z^{\prime}\right)=2 \pi \delta^{(2)}\left(z-z^{\prime}\right)$ and integrals of $\partial_{n} \tilde{G}$ over all the cycles $\partial \mathrm{D}_{1}, \ldots, \partial \mathrm{D}_{g}$ are zero. However, instead of being zero on the boundaries, $\tilde{G}$ takes there different constant values.

- $\tilde{\omega}_{\alpha}(z)$ : The modified harmonic measure is defined by

$$
\tilde{\omega}_{\alpha}(z)=-2 \sum_{\beta=1}^{g}\left(\Omega^{-1}\right)_{\alpha \beta} \omega_{\beta}(z)
$$

This is simply a linear combination of $\omega_{\alpha}$ 's with domain-dependent coefficients such that

$$
\frac{1}{2 \pi} \oint_{\partial \mathrm{D}_{\alpha}} \partial_{n} \tilde{\omega}_{\beta} d s=2 \delta_{\alpha \beta}
$$

The pressure field. Let us demonstrate how the solution for pressure in $D_{\text {oil }}$ is written in terms of the objects just introduced. For simplicity we do this assuming only one sink of oil with the power $Q$ located at a point $a(a=\infty$ is also possible).

The general solution for the pressure field $p=p(z)$ with $p=p_{\alpha}$ on the boundaries reads

$$
p(z)=2 Q G(z, a)+\sum_{\alpha=1}^{g} p_{\alpha} \omega_{\alpha}(z)=2 Q \tilde{G}(z, a)-\sum_{\alpha=1}^{g} q_{\alpha} \tilde{\omega}_{\alpha}(z)
$$

It is important to note that the rates $q_{\alpha}$ completely determine pressures $p_{\alpha}$ in the water droplets and vice versa. Indeed, plugging (37) into (3), we have the relation

$$
2 Q \oint_{\partial \mathrm{D}_{\alpha}} \partial_{n} G(a, z) d s+\sum_{\beta=1}^{g} p_{\beta} \oint_{\partial \mathrm{D}_{\alpha}} \partial_{n} \omega_{\beta}(z) d s=-4 \pi q_{\alpha}
$$


Using (33) and (34), we can write it either as a system of linear equations for $p_{\alpha}$ (in the $q$-representation),

$$
\frac{1}{2} \sum_{\beta=1}^{g} \Omega_{\alpha \beta} p_{\beta}=q_{\alpha}-Q \omega_{\alpha}(a)
$$

or a system of linear equations for $q_{\alpha}$ (in the $p$-representation),

$$
2 \sum_{\beta=1}^{g}(\Omega)_{\alpha \beta}^{-1} q_{\beta}=p_{\alpha}-Q \tilde{\omega}_{\alpha}(a)
$$

Since the $g \times g$ matrix $\Omega_{\alpha \beta}$ is non-degenerate, the system has a unique solution which is read from the equivalent "dual" system. Whichever the physical conditions in the water droplets are, pressure is given by

$$
p(z)=2 Q G(a, z)-2 Q \sum_{\alpha, \beta=1}^{g} \omega_{\alpha}(a)\left(\Omega^{-1}\right)_{\alpha \beta} \omega_{\beta}(z)+2 \sum_{\alpha, \beta=1}^{g} q_{\alpha}\left(\Omega^{-1}\right)_{\alpha \beta} \omega_{\beta}(z)
$$

Specifying this formula for the elementary processes, we have:

$$
p(z)=\left\{\begin{array}{ll}
2 G(a, z) & \text { for }(\mathrm{p} 1) \\
\omega_{\alpha}(z) & \text { for }(\mathrm{p} 2)
\end{array}, \quad p(z)= \begin{cases}2 \tilde{G}(a, z) & \text { for }(\mathrm{q} 1) \\
\tilde{\omega}_{\alpha}(z) & \text { for }(\mathrm{q} 2)\end{cases}\right.
$$

Variational formulas. Variations of the Green function and harmonic measures under infinitesimal deformations of the domain are described by remarkable formulas going back to Hadamard $[26,24]$. Let $\delta n(\xi)$ be the normal displacement (with sign) of the boundary under the deformation counted along the normal vector at the boundary point $\xi$, with the normal vector looking inside $\mathrm{D}_{\text {oil }}$, see Fig. 4 . The variational formulas are:

$$
\begin{aligned}
\delta G\left(z, z^{\prime}\right) & =\frac{1}{2 \pi} \oint_{\partial \mathrm{D}_{\text {oil }}} \partial_{n} G(z, \xi) \partial_{n} G\left(z^{\prime}, \xi\right) \delta n(\xi) d s \\
\delta \omega_{\alpha}(z) & =\frac{1}{2 \pi} \oint_{\partial \mathrm{D}_{\text {oil }}} \partial_{n} G(z, \xi) \partial_{n} \omega_{\alpha}(\xi) \delta n(\xi) d s \\
\delta \Omega_{\alpha \beta} & =\frac{1}{2 \pi} \oint_{\partial \mathrm{D}_{\text {oil }}} \partial_{n} \omega_{\alpha}(\xi) \partial_{n} \omega_{\beta}(\xi) \delta n(\xi) d s
\end{aligned}
$$

Small variations of the modified objects (with tilde) are described by exactly the same formulas (42), (43), where one should put tilde everywhere.

These variational formulas are easy to understand. Let us explain them on the example of the Green function. Since the Green function $G(z, \xi)$ vanishes if $\xi$ belongs to the old boundary, the variation $\delta G(z, \xi)$ is equal to the value of the new Green function on the old boundary, i.e., $\delta G(z, \xi)=-\delta n(\xi) \partial_{n} G(z, \xi)$, in the leading order. Now notice that $\delta G(z, \xi)$ is a harmonic function (the logarithmic singularity cancels since it is the same for both old and new functions) with the boundary value $-\delta n(\xi) \partial_{n} G(z, \xi)$. This function can be restored from its boundary value by solving the Dirichlet boundary problem. The r.h.s. of eq. (42) gives the result. 


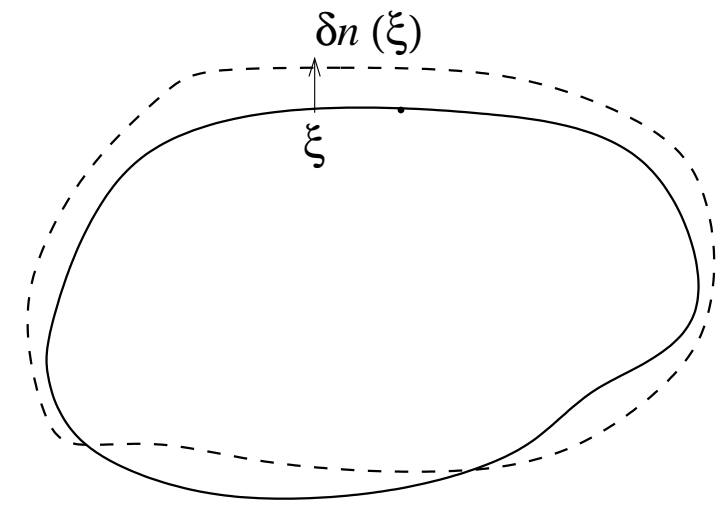

Figure 4: The normal displacement of the boundary.

When the domain evolves with time, the Green function and harmonic measures become time dependent. The variational formulas allow one to find time derivatives of these functions given a local law of motion of the boundary. This is the way how we derive partial differential equations for the ILG below.

\subsection{The Schottky double}

The growing domain $\mathrm{D}_{\text {oil }}$ is a Riemann surface with a border. From mathematical point of view, it is more convenient to work with compact Riemann surfaces without border than with bordered domains. Given a planar domain with holes, like $\mathrm{D}_{\text {oil }}$, endowed with the holomorphic coordinate $z$, it may be thought of as a "half" of a closed Riemann surface. Another half, an antiholomorphic "copy" of $\mathrm{D}_{\text {oil }}$ with coordinate $\bar{z}$, is glued to the first copy along the boundaries $\partial \mathrm{D}_{\alpha}$. Besides, each copy of $\mathrm{D}_{\text {oil }}$ should be compactified by adding a point at infinity. The resulting compact Riemann surface without boundary is called the Schottky double, or simply the double of the planar bordered domain (see, e.g. [24]).

More precisely, the Schottly double of a bordered surface, is a compact Riemann surface without boundary endowed with an antiholomorpic involution such that the boundary of the initial domain is the set of fixed points of the involution. The Schottky double of the $\mathrm{D}_{\text {oil }}$ can be thought of as two copies of it ("upper" and "lower" sheets of the double) glued along the boundaries $\cup_{\alpha=0}^{g} \partial \mathrm{D}_{\alpha}$, with points at infinity added ( $\infty$ and $\left.\bar{\infty}\right)$. The holomorphic coordinate on the upper sheet is $z$, the same as in $\mathrm{D}_{\text {oil }}$, while the holomorphic coordinate on the lower sheet is $\bar{z}$. The genus of the double is equal to the number of water droplets minus 1. The notion of the double was implicitly used by Richardson in his studies of viscous flows in multiply-connected fluid regions $[7,8]$.

A meromorphic function on the double is a pair of meromorphic functions $f, \tilde{f}$ in $\mathrm{D}_{\text {oil }}$ such that $f(z)=\tilde{f}(\bar{z})$ on the boundary. Similarly, a meromorphic differential on the double is a pair of meromorphic differentials $f(z) d z$ and $\tilde{f}(\bar{z}) d \bar{z}$ such that $f(z) d z=\tilde{f}(\bar{z}) d \bar{z}$ along the boundary curves. The Schwarz reflection principle says that any meromorphic differential $d \nu(z)$ on the upper sheet such that it is purely imaginary along the boundary, can be meromorphically extended to the lower sheet as $-\overline{d \nu(z)}$, so for each pole of such a globally 


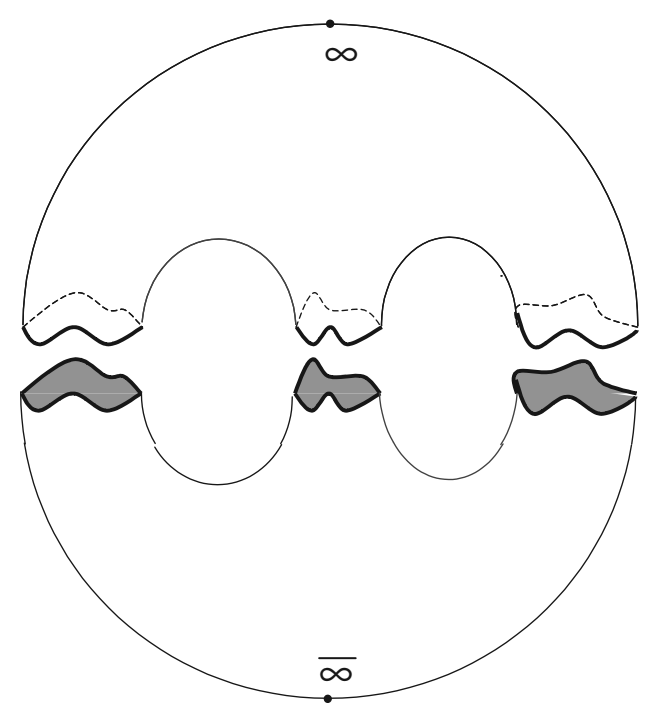

Figure 5: The two halfs of the Schottky double $(g=2)$.

defined differential there is a "mirror" pole on the opposite sheet.

To proceed, one has to choose a basis of $a-$ and $b$-cycles on the double having the canonical intersection form $a_{\alpha} \circ a_{\beta}=b_{\alpha} \circ b_{\beta}=0, a_{\alpha} \circ b_{\beta}=\delta_{\alpha \beta}$. In general, for an abstract Riemann surface, there is no preferred choice of the basis. However, when the surface is the double of a planar domain, like in our case, we may fix the following two distinguished ("dual") bases.

- The b-cycles are just boundaries of the holes $\mathrm{b}_{\alpha}=-\partial \mathrm{D}_{\alpha}$ for $\alpha=1, \ldots, g$. (Note, however, the negative clockwise orientation.) The $\mathrm{a}_{\alpha}$-cycle connects the boundary of the $\alpha$-th water droplet with the 0 -th one. To be more precise, fix points $\xi_{\alpha}$ on the boundaries, then the $a_{\alpha}$-cycle starts from $\xi_{0}$, goes to $\xi_{\alpha}$ on the "upper" sheet and returns following the same way on the "lower" sheet.

- In the "dual" basis, we interchange a- and b-cycles: $\tilde{a}_{\alpha}=-\mathrm{b}_{\alpha}, \tilde{\mathrm{b}}_{\alpha}=\mathrm{a}_{\alpha}$. The minus sign is necessary to preserve the anti-symmetric intersection form.

These two choices of the basic cycles correspond to the LG dynamics in the $p$ and $q$ representations.

\subsection{Differentials on the Schottky double}

Holomorphic differentials. Having fixed the basis of cycles, we can introduce normalized holomorphic differentials (abelian differentials of the first kind) as differentials of holomorphic parts of the harmonic measures. Let us represent $\omega_{\alpha}$ as the real part of a holomorphic function: $\omega_{\alpha}=W_{\alpha}(z)+\overline{W_{\alpha}(z)}$, where $W_{\alpha}(z)$ are holomorphic (in general multivalued) functions in $\mathrm{D}_{\text {oil }}$. The differentials $d W_{\alpha}=\partial_{z} \omega_{\alpha} d z$ are then holomorphic in $\mathrm{D}_{\text {oil }}$ and purely imaginary along all boundary contours. So they can be extended holomorphically to the lower 

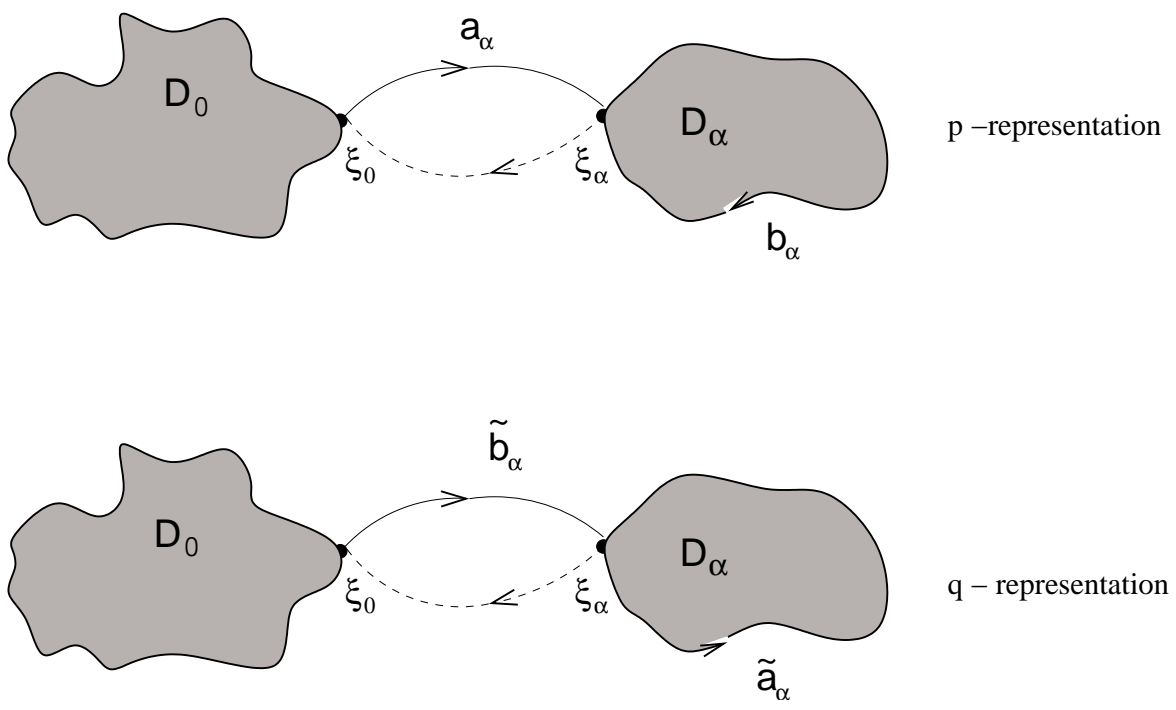

Figure 6: Canonical cycles on the Schottky double in $p$ and $q$-representations.

sheet as $-d \overline{W_{\alpha}(z)}$. They form the canonically normalized basis of holomorphic differentials on the double w.r.t. the a-cycles:

$$
\oint_{\mathbf{a}_{\alpha}} d W_{\beta}=\int_{\xi_{0}}^{\xi_{\alpha}} d W_{\beta}(z)+\int_{\xi_{\alpha}}^{\xi_{0}}\left(-d \overline{W_{\beta}(z)}\right)=2 \operatorname{Re} \int_{\xi_{0}}^{\xi_{\alpha}} d W_{\beta}(z)=\int_{\xi_{0}}^{\xi_{\alpha}} d \omega_{\beta}=\delta_{\alpha \beta}
$$

The matrix of b-periods of these differentials reads (cf. (34))

$$
T_{\alpha \beta}=\oint_{\mathbf{b}_{\alpha}} d W_{\beta}=-\frac{i}{2} \oint_{\mathbf{b}_{\alpha}} \partial_{n} \omega_{\beta} d s=i \pi \Omega_{\alpha \beta}
$$

Similarly, the differentials $d \tilde{W}_{\alpha}=\partial_{z} \tilde{\omega}_{\alpha} d z$, constructed in the same way from the modified harmonic measures, are normalized w.r.t. the ã-cycles ${ }^{1}$ :

$$
\oint_{\tilde{\mathrm{a}}_{\alpha}} d \tilde{W}_{\beta}=2 \pi i \delta_{\alpha \beta}
$$

The $\tilde{\mathbf{b}}$-periods of the differentials $d \tilde{W}_{\alpha}$ are:

$$
\oint_{\tilde{b}_{\alpha}} d \tilde{W}_{\beta}=-2\left(\Omega^{-1}\right)_{\alpha \beta}
$$

Meromorphic differentials [24]. The simplest meromorphic differential on a Riemann surface is the differential having only two simple poles with residues \pm 1 (the abelian differential of the third kind). We will especially need the differentials whose only simple poles are at the mirror points of the double. They are naturally associated with the oil pumps.

\footnotetext{
${ }^{1}$ Normalization of the holomorphic differentials differs by $2 \pi i$. One might work using the unified normalization in both $p$ and $q$-representations but in that case, as a price for the unified notation, some artificial imaginary units enter formulas for physical quantities.
} 
Specifically, consider the differential $d W^{(a, \bar{a})}(z)=2 \partial_{z} G(a, z) d z$ on the upper sheet. From the properties of the Green function it follows that this differential has the only simple pole with residue +1 at the point $a$. Since along all the boundaries

$$
0=d_{z} G(a, z)=\partial_{z} G(a, z) d z+\partial_{\bar{z}} G(a, z) d \bar{z}=2 \operatorname{Re}\left(d W^{(a, \bar{a})}(z)\right)=0
$$

this differential can be meromorphically extended to the lower sheet as $-2 \partial_{\bar{z}} G(a, z) d \bar{z}$, and so it has a simple pole with residue -1 at the mirror point $\bar{a}$ on the lower sheet. In particular, the differential $d W^{(\infty, \bar{\infty})}(z)=2 \partial_{z} G(\infty, z) d z$ has simple poles at the two infinite points of the Schottky double. The differentials $d \tilde{W}^{(a, \bar{a})}$ are constructed in the same way out of the modified Green function. Note that the so defined differential $d W^{(a, \bar{a})}$ are canonically normalized:

$$
\begin{array}{llll}
\oint_{\mathrm{a}_{\alpha}} d W^{(a, \bar{a})}=0 & \text { that means } & \operatorname{Re} \int_{\xi_{0}}^{\xi_{\alpha}} \partial_{z} G(a, z) d z=0 \quad(p \text {-representation }) \\
\oint_{\tilde{\mathrm{a}}_{\alpha}} d \tilde{W}^{(a, \bar{a})}=0 \quad \text { that means } \quad \oint_{\partial \mathrm{D}_{\alpha}} \partial_{z} G(a, z) d z=0 \quad \text { (q-representation) }
\end{array}
$$

The abelian differentials of the second kind, $d W_{k}^{(b)}$, have only one pole of order $k+1$ at the point $b$. These differentials can be explicitly defined by expansion of the Green function or the differential $d W^{(a, \bar{a})}$ in a Taylor series in $a-b$ in the vicinity of the point $b$. This differential is normalized, i.e., $\oint_{\mathrm{a}_{\alpha}} d W_{k}^{(a)}=0$ but to define it uniquely one has to fix the principal part at the pole.

Let us introduce the differentials $d W_{k}^{(\infty)}$ with the pole at infinity. Expanding the Green function around $\infty$ on the upper sheet, and using the basis of analytic functions $\psi_{k}$ (15), we write

$$
2 \partial_{z} G(a, z) d z=2 \partial_{z} G(\infty, z) d z-\sum_{k \geq 1} \frac{1}{k}\left(\psi_{k}(a) d W_{k}^{(\infty)}(z)+\overline{\psi_{k}(a)} d W_{k}^{(\bar{\infty})}(z)\right)
$$

Here $d W_{k}^{(\infty)}$ is the normalized differential holomorphic everywhere except infinity (on the upper sheet) with the principal part

$$
d W_{k}^{(\infty)}(z)=k d \chi_{k}(z)+O\left(z^{-2}\right) \quad \text { as } z \rightarrow \infty
$$

On the lower sheet, this differential is regular. Similarly, $d W_{k}^{(\bar{\infty})}$ has a pole at infinity on the lower sheet of the double and is regular on the upper sheet. The differentials are defined by the following contour integrals:

$$
d W_{k}^{(\infty)}=\left\{\begin{array}{cl}
\frac{k d z}{\pi i} \partial_{z} \oint_{\infty} \chi_{k}(\zeta) \partial_{\zeta} G(z, \zeta) d \zeta \quad \text { (on the upper sheet) } \\
-\frac{k d \bar{z}}{\pi i} \partial_{\bar{z}} \oint_{\infty} \chi_{k}(\zeta) \partial_{\zeta} G(z, \zeta) d \zeta \quad \text { (on the lower sheet) }
\end{array}\right.
$$

The contour here encircles $\infty$ but not the point $z$. The differential $d W_{k}^{(\bar{\infty})}$ is defined as the complex conjugated differential $\left(-d W_{k}^{(\infty)}\right)$ on the opposite sheet of the double.

The differentials introduced above are summarized in the table: 


\begin{tabular}{|c|c|c|c|c|}
\hline Type & Notation & $\begin{array}{l}\text { Explicit form on the up- } \\
\text { per/lower sheet }\end{array}$ & $\oint_{a_{\beta}}$ & $\oint_{b_{\beta}}$ \\
\hline \multirow[t]{2}{*}{ First kind } & \multirow[t]{2}{*}{$d W_{\alpha}$} & $\partial_{z} \omega_{\alpha}(z) d z$ & \multirow[t]{2}{*}{$\delta_{\alpha \beta}$} & \multirow[t]{2}{*}{$\pi i \Omega_{\alpha \beta}$} \\
\hline & & $-\partial_{\bar{z}} \omega_{\alpha}(z) d \bar{z}$ & & \\
\hline \multirow[t]{2}{*}{ Second kind } & \multirow[t]{2}{*}{$d W_{k}^{(\infty)}$} & see $(47)$ & \multirow[t]{2}{*}{0} & \multirow[t]{2}{*}{$k \oint_{\infty} d z \chi_{k}(z) \partial_{z} \omega_{\alpha}(z)$} \\
\hline & & see $(47)$ & & \\
\hline \multirow[t]{2}{*}{ Third kind } & \multirow[t]{2}{*}{$d W^{(a, \bar{a})}$} & $2 \partial_{z} G(a, z) d z$ & \multirow[t]{2}{*}{0} & \multirow[t]{2}{*}{$2 \pi i \omega_{\beta}(a)$} \\
\hline & & $-2 \partial_{\bar{z}} G(a, z) d \bar{z}$ & & \\
\hline
\end{tabular}

Correspondence between times and differentials. Each elementary flow is naturally "coupled" with a meromorphic or holomorphic differential on the Schottky double. This differential can be defined in terms of pressure as $\partial_{z} p(z) d z$. Equivalently it is a unique analytic continuation of the differential $\partial_{t} z(\sigma, t) d \bar{z}-\partial_{t} \overline{z(\sigma, t)} d z$ to $\mathrm{D}_{\text {oil }}$ from the boundary (here $\sigma$ is any parameter on the contours). The equivalence of the two definitions follows from the Laplacian growth equation (14) (Sec. 2.2). Explicitly, the coupling is:

$$
T^{(a)} \longmapsto d W^{(a, \bar{a})}, \quad T^{(\alpha)} \longmapsto d W_{\alpha}, \quad T_{k} \longmapsto d W_{k}^{(\infty)}
$$

In particular, $T^{(\infty)}=T_{0}+$ const, corresponding to an oil sink at infinity, is coupled with the differential $d W^{(\infty, \bar{\infty})}$. The abelian integral

$$
W(z)=W^{(\infty, \bar{\infty})}(z)=\int_{\zeta_{0}}^{z} d W^{(\infty, \bar{\infty})}
$$

where $\zeta_{0}$ is an arbitrary fixed point in $\mathrm{D}_{\text {oil }}$, has a constant real part along each component of the boundary and $W(z)=\log z+O(1)$ as $z \rightarrow \infty$. For the process (q1) with the sink at infinity the function $w(z)=e^{W(z)}$ conformally maps the domain $\mathrm{D}_{\text {oil }}$ onto the exterior of a disk of radius $r_{0}=\left|w\left(\xi_{0}\right)\right|\left(\xi_{0}\right.$ is any point on $\mathbf{b}_{0}$ ) with $g$ concentric arcs removed. Radii and endpoints of the arcs depend on time. The radius, $r_{\alpha}$, is related to pressure in the $\alpha$-water droplet by $p_{\alpha}=2 \log \left(r_{\alpha} / r_{0}\right)$.

\section{Equivalence of the ILG and Whitham dynamics}

We describe an evolution of the Riemann surface through a time dependence of the canonical abelian differentials and abelian integrals on the Schottky double. One may realize them as holomorphic or meromorphic functions of the coordinate $z$ on the physical plane. These functions encode the shape of the interface at any instant of time. In this respect they serve as substitutes for the time-dependent conformal map to the unit disk in case of a single droplet. 
We represent the dynamical equations of the ILG as relations between variations of the abelian integrals under simultaneous action of different oil and water pumps. Treating $T^{(a)}$ and $T^{(\alpha)}$ as an independent "time" variables the evolution takes the form of a hierarchy of partial differential equations in many variables. We recognize them as a universal Whitham hierarchy $[14,15]$ in the (extended) moduli space of genus $g$ Riemann surfaces (see Sec. 4.3).

\subsection{Exchange relations}

Let us calculate the time derivative of the Green function $G(a, b)$ in the elementary process with the oil pump at some third point $c$. Using the Hadamard formula (42), and the fact that for this process $\delta n(\xi)=-\frac{1}{2} \partial_{n} G(c, \xi) \delta T^{(c)}$, we have:

$$
\frac{\partial G(a, b)}{\partial T^{(c)}}=-\frac{1}{4 \pi} \oint_{\partial \mathrm{D}_{\mathrm{oil}}} \partial_{n} G(a, \xi) \partial_{n} G(b, \xi) \partial_{n} G(c, \xi) d s
$$

Remarkably, the result is symmetric under all permutations of the points $a, b, c$. For a single connected domain this equation has been obtained in Ref. [27]. In a similar way, we find, using (43), (44):

$$
\frac{\partial \omega_{\alpha}(b)}{\partial T^{(c)}}=-\frac{1}{4 \pi} \oint_{\partial \mathrm{D}_{\mathrm{oil}}} \partial_{n} \omega_{\alpha}(\xi) \partial_{n} G(b, \xi) \partial_{n} G(c, \xi) d s
$$

and

$$
\frac{\partial \Omega_{\alpha \beta}(b)}{\partial T^{(c)}}=-\frac{1}{4 \pi} \oint_{\partial \mathrm{D}_{\mathrm{oil}}} \partial_{n} \omega_{\alpha}(\xi) \partial_{n} \omega_{\beta}(\xi) \partial_{n} G(c, \xi) d s
$$

as well as analogous formulas for derivatives w.r.t. $T^{(\alpha)}$. Observing the symmetry of the right hand sides of these equations, one may write them as local relations connecting the time derivatives of the Green function and harmonic measures.

In this way we obtain the following fundamental exchange relations:

$$
\begin{gathered}
\frac{\partial G(a, b)}{\partial T^{(c)}}=\frac{\partial G(b, c)}{\partial T^{(a)}}=\frac{\partial G(c, a)}{\partial T^{(b)}} \\
\frac{\partial \omega_{\alpha}(a)}{\partial T^{(b)}}=\frac{\partial \omega_{\alpha}(b)}{\partial T^{(a)}} \\
\frac{\partial \omega_{\alpha}(a)}{\partial T^{(\beta)}}=\frac{\partial \omega_{\beta}(a)}{\partial T^{(\alpha)}}
\end{gathered}
$$

which encode dynamics of the Laplacian growth with zero surface tension. There is also the relation which connects derivatives of the Green function and harmonic measure:

$$
2 \frac{\partial G(a, b)}{\partial T^{(\alpha)}}=\frac{\partial \omega_{\alpha}(a)}{\partial T^{(b)}}
$$

In the same way, one may extend this list to include derivatives of the period matrix:

$$
2 \frac{\partial \omega_{\beta}(a)}{\partial T^{(\alpha)}}=\frac{\partial \Omega_{\alpha \beta}}{\partial T^{(a)}}, \quad \frac{\partial \Omega_{\alpha \beta}}{\partial T^{(\gamma)}}=\frac{\partial \Omega_{\beta \gamma}}{\partial T^{(\alpha)}}=\frac{\partial \Omega_{\gamma \alpha}}{\partial T^{(\beta)}}
$$

For the dual objects, the relations (49) - (52) remain the same. 


\subsection{The Whitham equations}

Whitham equations are holomorphic parts of the exchange relations. Taking, for instance, $\partial_{z}$ of both sides of the exchange relation $\partial_{T^{(b)}} G(a, z)=\partial_{T^{(a)}} G(b, z)$, we get $\partial_{T^{(b)}} d W^{(a, \bar{a})}(z)=$ $\partial_{T^{(a)}} d W^{(b, \bar{b})}(z)$ which is an equation of the Whitham hierarchy. The full list of Whitham equations obtained in this way reads:

$$
\begin{gathered}
\frac{\partial}{\partial T^{(a)}} d W^{(b, \bar{b})}(z)=\frac{\partial}{\partial T^{(b)}} d W^{(a, \bar{a})}(z) \\
\frac{\partial}{\partial T^{(\alpha)}} d W^{(a, \bar{a})}(z)=\frac{\partial}{\partial T^{(a)}} d W_{\alpha}(z) \\
\frac{\partial}{\partial T^{(\alpha)}} d W_{\beta}(z)=\frac{\partial}{\partial T^{(\beta)}} d W_{\alpha}(z)
\end{gathered}
$$

The derivatives are taken at constant $z$. The list can be further enlarged by adding the equations containing $T_{k}$-derivatives:

$$
\frac{\partial}{\partial T_{n}} d W_{k}^{(\infty)}(z)=\frac{\partial}{\partial T_{k}} d W_{n}^{(\infty)}(z)
$$

and so on. They can be obtained from the generating equations (54) - (56) by expending them in a series around infinity.

We note that there are a few other equivalent ways to write the Whitham equations [15]. Let us present a more invariant formulation, which does not rely on the choice of the distinguished local coordinate $z$ in $\mathrm{D}_{\text {oil }}$. The Whitham equations in the invariant form are naturally written in an extended "moduli space" of contours. Points of this extended space are sets of data of the form

$$
\left(g+1 \text { nonintersecting boundary contours; a point in } \mathrm{D}_{\text {oil }}\right)
$$

Local coordinates in this space are parameters $T \Phi$ or $T S$ and a local coordinate $\lambda$ in the domain $\mathrm{D}_{\text {oil }}$.

The local coordinate $\lambda$ may depend on the shape of the domain. In particular, one may choose $\lambda$ to be one of the abelian integrals, say $W(z)=W^{(\infty, \bar{\infty})}(z)$ with $\oint_{\mathrm{b}_{\alpha}} d W=0(48)$. Then the function $w(z)=e^{W(z)}$ is single-valued in $\mathrm{D}_{\text {oil }}$. It is a good local coordinate in $\mathrm{D}_{\text {oil }}$ everywhere except for the points where $d W^{(\infty, \bar{\infty})}=0$. It can be shown that all these points belong to the boundaries $b_{1}, \ldots, b_{g}$ and there are exactly two such points on each boundary. Under the conformal map $w(z)$ these points are taken to the endpoints of the concentric arcs (see the end of Sec.3.3). Treating all other abelian integrals as functions of $W$ rather than $z=z(W, T)$,

$$
W^{(a, \bar{a})}(W, T)=\int_{\zeta_{0}}^{z(W, T)} d W^{(a, \bar{a})}
$$

we represent the Whitham equations (54) - (56) in the form

$$
\frac{\partial W^{(A)}}{\partial T^{(B)}}-\frac{\partial W^{(B)}}{\partial T^{(A)}}+\left\{W^{(A)}, W^{(B)}\right\}=0
$$


where $A$ stands for $a$ or $\alpha$ and

$$
\left\{W^{(A)}, W^{(B)}\right\}:=\frac{\partial W^{(A)}}{\partial W} \frac{\partial W^{(B)}}{\partial T_{0}}-\frac{\partial W^{(B)}}{\partial W} \frac{\partial W^{(A)}}{\partial T_{0}}
$$

has the form of "Poisson brackets". These equations are consistency conditions for the system of evolution equations

$$
\frac{\partial z}{\partial T^{(A)}}=\left\{W^{(A)}, z\right\}
$$

Eqs. $(57,58)$ constitute the Whitham universal hierarchy of the soliton theory.

\subsection{Whitham equations in soliton theory}

Whitham equations as modulation equations. Integrable partial differential and difference equations of soliton theory are known to possess a rich family of periodic exact solutions depending on continuous parameters. To be more definite, we start our discussion with a $(1+1)$-dimensional integrable evolution equation of the form $u_{t}=P\left(u, u_{x}, \ldots\right)$ (e.g., the $\mathrm{KdV}$ equation). Exact periodic solutions have the form

$$
u(x, t)=u_{0}(\mathbf{U} x+\mathbf{V} t+\mathbf{Z} \mid I)
$$

where $\mathbf{U}, \mathbf{V}, \mathbf{Z}$ are $g$-dimensional constant vectors with components $\left\{U_{\alpha}\right\}=\left(U_{1}, \ldots, U_{g}\right)$, etc, and $u_{0}(\mathbf{Z})$ is a periodic function of any component $Z_{\alpha}$. This function, and all the vectors, depend on the set of parameters $I=\left(I_{1}, \ldots, I_{M}\right)$. Each periodic solution can be constructed starting from a Riemann surface. I stands for the moduli of a Riemann surface and the vectors $\mathbf{U}, \mathbf{V}$ are b-periods of certain normalized meromorphic differentials, $d W^{(x)}$ and $d W^{(t)}$, on the Riemann surface, with prescribed singularities at infinity [1].

In a number of physical problems one is interested in slowly modulated periodic solution, rather than just periodic. A nonlinear WKB method or the Whitham averaging method allows one to construct more general solutions of the same integrable equation using the function $u_{0}$ as a leading term of the asymptotic expansion

$$
u(x, t)=u_{0}\left(\varepsilon^{-1} \mathbf{S}(X, T)+\mathbf{Z}(X, T) \mid I(X, T)\right)+\varepsilon u_{1}(x, t)+\varepsilon^{2} u_{2}(x, t)+\ldots
$$

where $\varepsilon$ is a small parameter and the parameters $I$ now depend on the slow variables $X=\varepsilon x$, $T=\varepsilon t$. The original variables $x, t$ are called fast variables. If the vector-valued function $\mathbf{S}$ obeys the equations

$$
\partial_{X} \mathbf{S}=\mathbf{U}(I(X, T)), \quad \partial_{T} \mathbf{S}=\mathbf{V}(I(X, T))
$$

then the leading term agrees with the original solution up to first order in $\varepsilon$. All the higher corrections can be found by solving non-homogeneous linear equations whose homogeneous part is the original equation linearized on the background of the exact solution $u_{0}$ [12].

We see that the so constructed solution $u(x, t)$ describes the original fast periodic oscillations, modulated, on a larger scale, by a slow drift in the space of exact periodic solutions. The equation, which describes the drift $I(X, T)$ are called Whitham equations. For the 
particular example discussed above the equation to determine $I(X, T)$, written in a proper local parameter reads $\partial_{X} d W^{(t)}=\partial_{T} d W^{(x)}$. This form of the Whitham equations was first observed in [13] for the KdV hierarchy. Implicitly, through the dependence of the canonically normalized differentials on the slow variables, they describe the drift in the moduli space of Riemann surfaces and thus the dependence $I(X, T)$. The Hamiltonian approach to Whitham equations for $(1+1)$-dimensional systems was developed in [28]. A universal Whitham hierarchy in a general setting of multi-dimensional integrable equations was suggested in ref. [14]. An invariant formulation of the Whitham hierarchy, independent on the choice of local coordinates, was given in [16].

The idea of [14] was to obtain equations describing the slow drift in the space of exact solutions from the condition that next-to-leading terms of the asymptotic series $u(x, t)$ be uniformly bounded on large scales. In general, the asymptotic series becomes unreliable on scales of order $\varepsilon^{-1}$, i.e., the corrections become large. The main result of [14] is that the Whitham equations follow if one requires that just the next term of the series, $u_{1}(x, t)$, be uniformly bounded for all $x, t$.

The universal Whitham hierarchy. In a more general multi-dimensional hierarchy of soliton equations (like the KP hierarchy or the 2D Toda lattice hierarchy, or their difference counterparts), one has a family of "times" $t_{A}$ and a family of "potentials" $u^{(N)}$, evolving with times $t_{A}$. (Here $A$ and $N$ belong to a case-dependent, generally infinite set of indices.)

Exact periodic solutions of the hierarchy are constructed from a given time-independent Riemann surface $\Gamma$ with some additional data on it. With each time $t_{A}$ one associates a meromorphic differential, $d W_{A}$, on $\Gamma$ normalized with respect to, say, a-cycles: $\oint_{\mathrm{a}_{\alpha}} d W_{A}=0$. Let $\mathbf{U}^{(A)}$ be the vector of b-periods of this differential:

$$
U_{\alpha}^{(A)}=\oint_{\mathbf{b}_{\alpha}} d W_{A}
$$

Then the exact solution has the form ( $u$ is one of $u^{(N)}$ 's):

$$
u\left(\left\{t_{A}\right\}\right)=u_{0}\left(\sum_{A} \mathbf{U}^{(A)}(I) t_{A}+\mathbf{Z}(I) \mid I\right)+c_{0}(I)
$$

where $u_{0}$ is a certain oscillating periodic function (the second logarithmic derivative of the Riemann $\theta$-function) and $c_{0}$ is a constant. Like in the previous example, one may try to construct a more general oscillating, but not periodic solutions with slowly varying parameters

$$
u=u_{0}\left(\varepsilon^{-1} \mathbf{S}\left(\left\{T_{A}\right\}\right)+\mathbf{Z}\left(\left\{T_{A}\right\}\right) \mid I\left(\left\{T_{A}\right\}\right)\right)+c_{0}\left(I\left(\left\{T_{A}\right\}\right)\right)+\varepsilon u_{1}\left(\left\{t_{A}\right\}\right)+\ldots
$$

Here $T_{A}=\varepsilon t_{A}$ are slow times and $\mathbf{S}$ is a vector function such that $\partial_{T_{A}} \mathbf{S}=\mathbf{U}^{(A)}\left(I\left(\left\{T_{A}\right\}\right)\right)$. The uniform boundness of the first correction $u_{1}$ for all times implies the hierarchy of Whitham equations [15],

$$
\frac{\partial W_{A}}{\partial \lambda}\left(\frac{\partial W_{B}}{\partial T_{C}}-\frac{\partial W_{C}}{\partial T_{B}}\right)+\frac{\partial W_{B}}{\partial \lambda}\left(\frac{\partial W_{C}}{\partial T_{A}}-\frac{\partial W_{A}}{\partial T_{C}}\right)+\frac{\partial W_{C}}{\partial \lambda}\left(\frac{\partial W_{A}}{\partial T_{B}}-\frac{\partial W_{B}}{\partial T_{A}}\right)=0
$$


valid for all possible values of the indices $A, B, C$. Here $\lambda$ is any local parameter, all the abelian integrals being regarded as functions of $\lambda$. Choosing one of the indices, say $C$, to be $(\infty, \bar{\infty})$ and $\lambda=W=W^{(\infty, \bar{\infty})}$, one gets the Whitham equations in the form (57).

While averaging the solution (59) over fast oscillations, $\left\langle u_{0}\right\rangle$ vanishes

$$
\langle u\rangle\left(\left\{T_{A}\right\}\right)=c_{0}\left(\left\{T_{A}\right\}\right)
$$

In the context of Laplacian growth, $T_{A}$ is $T^{(a)}$ or $T^{(\alpha)}$, and $c_{0}$ is the Green function $G(a, b)$. Here the points $(a, b)$ label the potential. Thus the Laplacian growth can be thought of as a physical realization of the slow drift in the moduli space of Riemann surfaces.

\section{Special classes of solutions of the Whitham hierarchy and Laplacian growth of algebraic domains}

In this section we briefly discuss particularly important families of growing domains. They correspond to special solutions to the Whitham hierarchy called "algebraic orbits" [15].

\subsection{Algebraic and abelian domains}

Let us recall that the time derivative of the Cauchy transform of the oil domain (10) is a globally defined rational function. Consider the class of domains whose Cauchy transform is a single globally defined meromorphic (i.e., rational) function in the plane. In other words, each function $h_{\alpha}^{+}$defined originally in the domain $D_{\alpha}$ is extendable to a single rational function defined everywhere in the plane, same for different $\alpha$. In this case one may forget about the index $\alpha$ and deal with the single function $h(z)$. Eq. (10) tells us that if the initial fluid region is from this class, then it remains to be in this class in the process of the LG evolution. The evolution may only add new poles or change residues of the existing ones. As is pointed out in [8], in order to prepare such an initial condition, one may inject oil through points into a cell initially filled by water.

The domains whose Cauchy transform is a globally defined rational function are called algebraic [11] or quadrature domains [29]. Some illustrative examples in the multiply-connected case can be found in [8] and [30]. This class appears to be quite representative and important since any domain with smooth boundary components can be approximated by quadrature domains (see [31] for the proof). In the simply-connected case, the quadrature domains are images of the unit disk under conformal maps given by rational functions. Their time evolution is described by rational solutions of the Laplacian growth equation. These solutions are sometimes known to develop cusp-like singularities within finite time [19].

A more general class of domains can be defined by imposing the above condition not on the $h_{\alpha}^{+}$itself but on its $z$-derivative. Namely, suppose that each differential $d h_{\alpha}^{+}(z)$ is extendable to a meromorphic differential in the plane, and they coincide for different $\alpha$ 's. In this case $h^{+}$itself may be a multivalued analytic function with logarithmic branch points. In [11], such domains were called abelian domains. They can be produced from the quadrature 
domains by the oil sucking from linearly extended sinks. In the simply-connected case, their evolution is described by logarithmic solutions of the Laplacian growth equation [32, 33].

\subsection{The Schwarz function}

For domains with analytic boundaries, and for algebraic domains in particular, the Cauchy transform allows one to introduce the Schwarz function of the boundary contours, which proved to be very useful for analyzing the LG dynamics in the simply-connected case [2]. Given a closed contour on the plane, the Schwarz function [29, 34] is defined as the analytic continuation of the function $\bar{z}$ away from the contour. Let us denote it by $S(z)$. According to the definition, $S(z)$ is a function analytic in some neighborhood of the curve such that

$$
S(z)=\bar{z} \quad \text { on the curve }
$$

From the continuity of the Cauchy transform we have $\bar{z}=h_{\alpha}^{+}(z)-h^{-}(z)$ on the $\alpha$-th boundary, so $S^{(\alpha)}(z)=h_{\alpha}^{+}(z)-h^{-}(z)$ is the Schwarz function of the $\alpha$-th boundary curve. In general all these functions are different. However, as it directly follows from the definition, all the boundary contours of algebraic or abelian domains have a common Schwarz function, $S(z)=S^{(\alpha)}(z)$ for any $\alpha$, and the differential $d S(z)$ is meromorphic in $\mathrm{D}_{\text {oil }}$, i.e., it has there only a finite number of isolated poles.

In the case of algebraic or abelian domains, one may decompose the Schwarz function into the sum $S(z)=S_{+}(z)+S_{-}(z)$, where the function $S_{+}=h_{\alpha}^{+}(z)$ (for any $\alpha$ ) is analytic inside water droplets while $S_{-}=-h^{-}(z)$ is analytic in $\mathrm{D}_{\text {oil }}$ and vanishes at infinity. Combining the time derivatives of $h_{\alpha}^{+}$and $h^{-}$(see (8) and (9)), we find the time derivative $\dot{S}(z)=\partial_{t} S(z ; t)$ of the Schwarz function at constant $z$ :

$$
\dot{S}(z)=\dot{h}_{\alpha}^{+}(z)-\dot{h}^{-}(z)=-\partial_{z} p(z) \quad\left(\text { for } z \text { in } \mathrm{D}_{\mathrm{oil}}\right)
$$

where by $h_{\alpha}(z)$ we mean the analytic continuation of this function to $D_{\text {oil }}$.

In $\mathrm{D}_{\alpha}$ 's, the analytically continued function $S(z)$ has more complicated singularities. In case of general position, they are branch points $\eta_{i}$ of order two (with cuts between them). One may think of the algebraic curve underlying the solution to the LG problem as the Riemann surface of the function $S(z)$. The Whitham equations can then be equivalently represented in the form of equations for the endpoints of the cuts, which describe their time dependence $\eta_{i}=\eta_{i}(T)$. The equations are:

$$
\frac{\partial \eta_{i}}{\partial T^{(A)}}=\left(\frac{d W^{(A)}(z)}{d W(z)}\right)_{z=\eta_{i}} \frac{\partial \eta_{i}}{\partial T_{0}}
$$

where the coefficients in front of $\partial \eta_{i} / \partial T_{0}$ in the r.h.s. are expressible as (in general quite complicated) functions of the $\eta_{k}$ 's. Basically these equations mean that the Schwarz function takes finite values at the branch points $\eta_{i}$. 


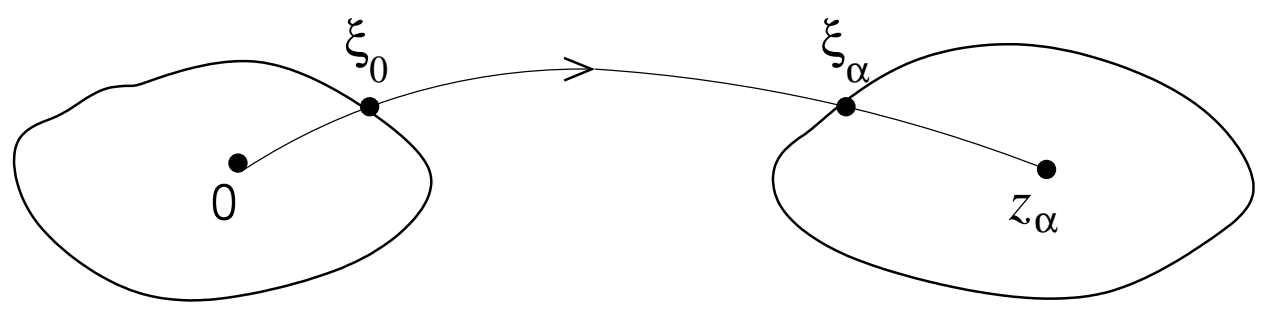

Figure 7: The integration path from $z_{0}=0$ to $z_{\alpha}$.

\subsection{The generating differential}

For the class of algebraic domains, one may introduce a distinguished meromorphic differential on the Schottky double. Recall that the Schwarz function $S(z)$ is a meromorphic function in $\mathrm{D}_{\text {oil }}$, if $\mathrm{D}_{\text {oil }}$ is an algebraic domain. Therefore, one may treat $S(z)$ as a function on the Schottky double extending it to the lower sheet as $\bar{z}$. In this case the differential $d \mathcal{S}=S(z) d z$ is extendable to a meromorphic differential on the double. Its explicit form on the lower sheet is $\bar{z} \overline{S(z)}$.

The differential $d \mathcal{S}$ plays an important role in the theory. We call it the generating differential because it generates the complete set of local coordinates in the space of algebraic multiply-connected domains. Indeed, the harmonic moments are

$$
T_{k}=-\frac{1}{2 \pi i k} \oint_{\partial \mathrm{D}_{\mathrm{oil}}} \psi_{k}(z) S(z) d z
$$

The areas of water droplets are periods of this differential over b-cycles:

$$
S_{\alpha}=-\frac{1}{2 \pi i} \oint_{\mathrm{b}_{\alpha}} d \mathcal{S}
$$

The "dual" variables, $\Phi_{\alpha}$, are expressed through periods of the same differential over the a-cycles (or, what is the same, over $\tilde{b}$-cycles). To show this, we write:

$$
\begin{gathered}
\Phi_{\alpha}=\int_{0}^{z_{\alpha}} d \phi(z)=2 \operatorname{Re} \int_{0}^{z_{\alpha}} h(z) d z= \\
=2 \operatorname{Re}\left(\int_{0}^{\xi_{0}} h_{0}^{+}(z) d z+\int_{\xi_{\alpha}}^{z_{\alpha}} h_{\alpha}^{+}(z) d z+\int_{\xi_{0}}^{\xi_{\alpha}}\left(\bar{z}+h^{-}(z)\right) d z\right)
\end{gathered}
$$

where the integral from 0 to $z_{\alpha}$ goes along a path containing the two points and $\xi_{0}, \xi_{\alpha}$ are intersection points of the path with the boundary curves $b_{0}, b_{\alpha}$ (see Fig. 7). Adding and subtracting $\int_{\xi_{0}}^{\xi_{\alpha}} h^{+}(z) d z$ (which is well-defined for algebraic domains), we rewrite this in the form

$$
\Phi_{\alpha}=2 \operatorname{Re} \int_{0}^{z_{\alpha}} S_{+}(z) d z-\Pi_{\alpha}
$$

where

$$
\Pi_{\alpha}=2 \operatorname{Re} \int_{\xi_{0}}^{\xi_{\alpha}}(S(z)-\bar{z}) d z=\int_{\xi_{0}}^{\xi_{\alpha}}(S(z) d z-\bar{z} d \overline{S(z)})=\oint_{\mathrm{a}_{\alpha}} d \mathcal{S}
$$


Note that $\Pi_{\alpha}$ does not depend on the points $\xi_{0}, \xi_{\alpha}$. Finally, the times $T^{(a)}$ associated with a sink of oil at the point $a$ are expressed as

$$
T^{(a)}=-\frac{1}{2 \pi i} \oint_{c_{a}} d \mathcal{S}
$$

(Here $\mathrm{c}_{a}$ is a small contour encircling the point $a$.)

The table of differentials from Sec. 3.3 can be continued by including the generating differential:

\begin{tabular}{|l|c|c|c|c|}
\hline Type & Notation & $\begin{array}{l}\text { Explicit form on the up- } \\
\text { per/lower sheet }\end{array}$ & $\oint_{\mathrm{a}_{\alpha}}$ & $\oint_{\mathrm{b}_{\alpha}}$ \\
\hline $\begin{array}{l}\text { Generating } \\
\text { differential }\end{array}$ & $d \mathcal{S}$ & $S(z) d z$ & $\Pi_{\alpha}$ & $-2 \pi i S_{\alpha}$ \\
\cline { 3 - 5 } & $\bar{z} d \overline{S(z)}$ & & \\
\hline
\end{tabular}

Moreover, partial derivatives of the generating differential w.r.t. the times $T^{(a)}, T^{(\alpha)}$ coincide with the canonical meromorphic differentials:

$$
\frac{\partial S(z)}{\partial T^{(a)}} d z=-d W^{(a, \bar{a})}(z), \quad \frac{\partial S(z)}{\partial T^{(\alpha)}} d z=-d W_{\alpha}(z)
$$

This follows from (62) after substituting the pressure filed for the elementary processes in terms of the Green function and harmonic measures (see (41)). For algebraic domains, the Whitham equations follow from the existence of the generating differential and (68).

The generating differential can be represented as

$$
d \mathcal{S}=d \Lambda-\sum_{A} T^{(A)} d W^{(A)}
$$

where $d \Lambda$ is a differential with $T^{(A)}$-independent singularities. For algebraic orbits, it is a fixed meromorphic differential (possibly with time-independent jumps). In more general cases $d \Lambda$ has more complicated analytic properties. Presumably, it can be defined as a solution to a $\bar{\partial}$-problem. The expansion (69) and equation $d \mathcal{S}\left(\lambda_{s}\right)=0$ for all zeros $\lambda_{s}$ of the differential $d z$ on the lower sheet of the Schottky double, where it has the form $d \overline{S(z)}$, are key relations which imply (68). Indeed, from the latter condition it follows that the differential $\partial_{A} d \mathcal{S}$ has no singularities at the points $\lambda_{s}$. Then (69) implies that this differential has the same singularities and periods as $-d W^{(A)}$. Hence, they do coincide.

General algebraic orbits of Whitham equations for higher genus Riemann surfaces in the sense of [15] correspond to the case when the z-derivative of the Schwarz function extends to a meromorphic function on the double (equivalently, when the differential $d S(z)$ extends to a meromorphic differential on the double). 


\section{Conclusion}

In short, the main message of this work is that the variables in which the Laplacian growth with zero surface tension becomes linear, for arbitrary connectivity of the growing domain and arbitrary configuration of pumps, are the Whitham "times" defined in [15]. The latter are special local coordinates on the extended moduli space of Riemann surfaces. Conservation or linear dependence on time of harmonic moments of the growing domain, known before as a characteristic feature of the idealized Laplacian growth, is a particular case of this result.

The Whitham equations are partial differential equations for canonical holomorphic and meromorphic differentials on Riemann surfaces regarded as functions of the local coordinates in the moduli space. Solutions to the Whitham equations allow one to find the differentials and abelian integrals as functions of time and reconstruct dynamics of the interface.

The Whitham equations are often regarded as integrable ones, though not in the Liouville sense. When speaking about integrability of Whitham equations, one means mainly a possibility to actually integrate them by representing a solution in the form of an implicit function of independent variables (the hodograph method).

The Whitham equations appear in soliton theory in different contexts. First, when one looks for solutions of soliton equations other than periodic. At some regimes, these solutions are well approximated, on small space-time scales, by the periodic exact solutions of the algebro-geometric type. When fast oscillations of the periodic solutions are averaged or smoothed out, Whitham equations appear as modulation equations written for moduli of the Riemann surface parametrizing the algebro-geometric periodic solutions. An important special case of Whitham equations appears if one neglects dispersion in nonlinear soliton equations. The latter case provides the most direct link to the Laplacian growth of simplyconnected domains. This link was explored in Ref.[2]. What is perhaps the most important conclusion, the Whitham equations describe a proper evolution of the Riemann surface built upon a growing interface.

The relation between the growth problem and modulated periodic solutions to soliton equations is two-fold. The Laplacian growth may serve as a simple illustrative physical model of the Whitham dynamics of complex curves. Vice versa, the methods developed in soliton theory may help to understand growth in a singular (turbulent) regime, i.e., in a vicinity of cusp formation or coalescence and break-up of droplets, providing an effective account of the surface tension effects near singular points.

\section{Acknowledgments}

We are indebted to O.Agam, E.Bettelheim, V.Kazakov, A.Marshakov, R.Theodorescu for useful discussions and N.Amburg for help with figures. The work of I.K. was supported by NSF grant DMS-01-04621. M.M.-W. and A.Z. were supported by the LDRD project 20020006ER "Unstable Fluid/Fluid Interfaces" at Los Alamos National Laboratory. P. W. was supported by the NSF MRSEC Program under DMR-0213745, NSF DMR-0220198 and by Humboldt foundation. The work of A.Z. was also supported in part by RFBR grant 03- 
02-17373 and by grant for support of scientific schools NSh-1999.2003.2. A.Z. is grateful to CNLS at Los Alamos National Laboratory, where this work was completed, for hospitality.

\section{References}

[1] S.P.Novikov, S.V.Manakov, L.P.Pitaevski and V.E.Zakharov, Theory of Solitons: The Inverse Scattering Method, Plenum Press, 1984

[2] M.Mineev-Weinstein, P.Wiegmann and A.Zabrodin, Phys. Rev. Lett. 84 (2000) 5106

[3] K.A.Gillow and S.D.Howison, A bibliography of free and moving boundary problems for Hele-Shaw and Stokes flow, http://www.maths.ox.ac.uk/ howison/Hele-Shaw/

[4] D.Bensimon, L.P.Kadanoff, S.Liang, B.I.Shraiman and C.Tang, Rev. Mod. Phys. 58 (1986) 977-999

[5] E.Ikeda and T.Maxworthy, Phys. Rev. A 41 (1990) 4367-4370;

M.G.Moore, A.Juel, J.M.Burgess, W.D.McCormick and H.L.Swinney, AIP Conference Proceedings 676 (2003) 189-194; The 7th Experimental Chaos Conference; Aug. 26-29, 2002; San Diego, CA

[6] S.Richardson, J. Fluid Mech. 56 (1972) 609-618

[7] S.Richardson, Euro. J. of Appl. Math. 5 (1994) 97-122;

Phil. Trans. R. Soc. London A 354 (1996) 2513-2553

[8] S.Richardson, Euro. J. of Appl. Math. 12 (2001) 571-599

[9] P.Etingof, Dokl. Akad. Nauk SSSR 313 (1990) 42-47

[10] D.G.Crowdy and S.Tanveer, J. Nonlinear Sci. 8 (1998) 375-400;

D.G.Crowdy and H.Kang, J. Nonlinear Sci. 11 (1998) 279-304;

D.Crowdy, SIAM J. Appl. Math. 62 (2002) 945-964

[11] P.Etingof and A.Varchenko, Why does the boundary of a round drop becomes a curve of order four, University Lecture Series, 3, American Mathematical Society, Providence, RI, 1992

[12] G.B.Whitham, Linear and nonlinear waves, Wiley Interscience, New York, 1974

[13] H.Flashka, M.G.Forest and D.McLaughlin, Comm. Pure Appl. Math. 33 (1980) 739

[14] I.M.Krichever, Funct. Anal Appl. 22 (1989) 200-213

[15] I.M.Krichever, Comm. Pure. Appl. Math. 47 (1992) 437-476

[16] I.M.Krichever, Russian Math. Surveys, 44 (1989) 145-225

[17] Singular Limits of Dispersive Waves, ed. N.M.Ercolani et al., Plenum Press, NY, 1994 
[18] A.Gurevich and L.Pitaevskii, Sov. Phys. JETP 38:2 (1974) 291-297;

B.Dubrovin and S.P.Novikov, Russian Math. Surveys 44:6 (1989) 35-124

[19] B.Shraiman and D.Bensimon, Phys. Rev. A 30 (1984) 2840-2842

[20] L.A.Galin, Dokl. Akad. Nauk SSSR 47 (1945) 250-253;

P.Ya.Polubarinova-Kochina, Dokl. Akad. Nauk SSSR, 47 (1945) 254-257;

P.P.Kufarev, Dokl. Akad. Nauk SSSR 57 (1947) 335-348

[21] I.Krichever and S.Novikov, Funk. Analiz i ego Pril. 21 (1987) No 2 46-63

[22] I.Krichever, A.Marshakov and A.Zabrodin, Integrable Structure of the Dirichlet Boundary Problem in Multiply-Connected Domains, Commun. Math. Phys. (2004), to be published, e-print archive: hep-th/0309010

[23] P.S.Novikov, Soviet Math. Dokl., 18 (1938) 165-168;

L.Zalcman, Contemp. Math. 63 (1987) 337-349

[24] M.Schiffer and D.C.Spencer, Functionals of finite Riemann surfaces, Princeton University Press, 1954

[25] F.Gakhov, The boundary value problems, Nauka, Moscow, 1977 (in Russian)

[26] J.Hadamard, Mém. présentés par divers savants à l'Acad. sci., 33 (1908).

[27] A.Marshakov, P.Wiegmann and A.Zabrodin, Commun. Math. Phys. 227 (2002) 131, e-print archive: hep-th/0109048.

[28] B.A.Dubrovin and S.P.Novikov, Soviet Math. Dokl., 27 (1983) 665-669

[29] D.Aharonov and H.Shapiro, J. Anal. Math. 30 (1976) 39-73;

H.Shapiro, The Schwarz function and its generalization to higher dimensions, University of Arkansas Lecture Notes in the Mathematical Sciences, Volume 9, W.H. Summers, Series Editor, A Wiley-Interscience Publication, John Wiley and Sons, 1992

[30] D.Crowdy, Proc. R. Soc. Lond. A (2001) 457 2337-2359;

D.Crowdy and J.Marshall, SIAM J. Appl. Math. 64 (2004) 1334

D. Crowdy, "Quadrature domains and fluid dynamics", to appear in a book "Quadrature domains and applications", eds M. Putinar and B. Gustafsson, Birkhauser, (2004).

[31] B.Gustafsson, Acta Applicandae Mathematicae 1 (1983) 209-240

[32] S.D.Howison, J. Fluid Mech. 167 (1986) 439-453

[33] M.Mineev-Weinstein and S.P.Dawson, Phys. Rev. E 50 (1994) R24-R27;

S.P.Dawson and M.Mineev-Weinstein, Physica D 73 (1994) 373-387

[34] P.J.Davis, The Schwarz function and its applications, The Carus Math. Monographs, No. 17, The Math. Assotiation of America, Buffalo, N.Y., 1974 